\title{
Stochastic Orderings of Multivariate Elliptical Distributions
}

\author{
Chuancun Yin \\ School of Statistics, Qufu Normal University \\ Shandong 273165, China \\ e-mail: ccyin@qfnu.edu.cn
}

November 27, 2019

\begin{abstract}
Let $\mathbf{X}$ and $\mathbf{Y}$ be two $n$-dimensional elliptical random vectors, we establish an identity for $E[f(\mathbf{Y})]-E[f(\mathbf{X})]$, where $f: \mathbb{R}^{n} \rightarrow \mathbb{R}$ satisfies some regularity conditions. Using this identity we provide a unified derivation of sufficient and necessary conditions for classifying multivariate elliptical random vectors according to several main integral stochastic orders. As a consequence we obtain new inequalities by applying it to multivariate elliptical distributions. The results generalize the corresponding ones for multivariate normal random vectors in the literature.
\end{abstract}

Keywords: Increasing convex order; multivariate elliptical distribution; multivariate normal distribution; supermodular order; usual stochastic order

AMS 2000 subject classifications: 60E15

\section{Introduction}

Stochastic orders provide methods of comparing random variables and vectors which are now used in many areas such as statistics and probability (Cal and Carcamo (2006), Hu and Zhuang (2006), Müller and Scarsini (2006), Fill and Kahn (2013) and Hazra et al. (2017)), operations research (Fábián et al. (2011)), actuarial sciences and economic theory (Baüerle and Bayraktar (2014), López-Díaz et al. (2018)), risk management and other related fields (Bäuerle and Müller (2006)). For a comprehensive review of the properties and characterizations of stochastic orderings, including a variety of applications, the reader is referred to the monographs of Müller and Stoyan (2002), Denuit et al. (2005), and Shaked and Shanthikumar (2007). Many of these orders are characterized by the so-called integral stochastic orders which is obtained by comparing expectations of functions in a certain class. A general treatment for these orders has been given in Whitt (1986) and Müller (1997). 
Elliptical distributions are generalizations of the multivariate normal distributions and, therefore, share many of the tractable properties. This class of distributions was introduced by Kelker (1970) and was extensively discussed in Fang et al. (1987). This generalization of the normal family seems to provide an attractive tool for statistics, economics and finance, which can describe fat or light tails and tail dependence among components of a vector. Interested readers are referred to monograph of Gupta, Varga and Bodnar (2013) and some recent papers of El Karoui (2009), Hu et al. (2019) and Sha et al. (2019). Müller (2001) studied the stochastic ordering characterizations of multivariate normal random vectors. Arlotto and Scarsini (2009) unified and generalized several known results on comparisons of multivariate normal random vectors in the sense of different stochastic orders by introducing the socalled Hessian order. Landsman and Tsanakas (2006) derived necessary and sufficient conditions for classifying bivariate elliptical distributions through the concordance ordering. Ding and Zhang (2004) extended the results in Müller (2001) to Kotz-type distributions which form an important specially class of elliptical symmetric distributions. Necessary and sufficient conditions for convex order and increasing convex order of general multivariate elliptical random vectors had not been found until the work of Pan et al. (2016). However, few results can be found in the literature that characterize the supermodular order of multivariate elliptical distributions. It is the aim of this paper to fill this gap. We will give some sufficient and necessary conditions for supermodular order of multivariate elliptical random vectors. For the known results such as on the convex ordering and the increasing convex ordering of multivariate elliptical random vectors, we provide a different simple proof.

The rest of the paper is organized as follows. Section 2 recalls some useful notions that will be used in the sequel, such as certain properties of stochastic orders and elliptical distributions. Section 3 presents necessary and sufficient conditions for several important stochastic orders of multivariate elliptical distributions. Section 4 provides two applications of the main results.

\section{Preliminaries}

Throughout this paper we use the following notations. We use bold letters to denote vectors or matrices. For example, $\mathbf{X}^{\prime}=\left(X_{1}, \cdots, X_{n}\right)$ is a row vector and $\boldsymbol{\Sigma}=\left(\sigma_{i j}\right)_{n \times n}$ is an $n \times n$ matrix. In particular, the symbol $\mathbf{0}_{n}$ denotes the $n$-dimensional column vector with all entries equal to $0, \mathbf{1}_{n}$ denotes the $n$-dimensional column vector with all components equal to 1 , and $\mathbf{1}_{n \times n}$ denotes the $n \times n$ matrix with all entries equal to 1 . Denote by $\mathbf{O}_{n \times n}$ the $n \times n$ matrix with all entries equal to 0 and by $\mathbf{I}_{n}$ the $n \times n$ identity matrix. For symmetric matrices $A$ and $B$ of the same size, the notion $A \preceq B$ or $B-A \succeq \mathbf{O}$ means that $B-A$ is positive semi-definite. The inequality between vectors or matrices denotes componentwise inequalities. Throughout this paper, the terms of increasing and decreasing are used in the weak sense. All integrals and expectations are implicitly assumed to exist whenever they appear. 


\subsection{Stochastic orders}

In this section, we summarize some important definitions and facts about the stochastic orderings of random vectors. The standard references for stochastic orderings are the monographs by Denuit et al. (2005) and Shaked and Shanthikumar (2007). For a function $f: \mathbb{R}^{n} \rightarrow \mathbb{R}, \mathbf{x} \in \mathbb{R}^{n}, i \in\{1, \cdots, n\}$ and $\delta>0$, we define the difference operator $\Delta_{i}^{\delta}$ as

$$
\Delta_{i}^{\delta} f(\mathbf{x})=f\left(\mathbf{x}+\delta \mathbf{e}_{i}\right)-f(\mathbf{x}),
$$

where $\mathbf{e}_{i}=(0, \ldots, 0,1,0, \ldots, 0)$ denotes the $i$ th unit vector. In case $n=1$ we simply write

$$
\Delta^{\delta} f(x)=f(x+\delta)-f(x) .
$$

A function $f: \mathbb{R}^{n} \rightarrow \mathbb{R}$ is said to be increasing, if $\Delta_{i}^{\delta} f(\mathbf{x}) \geq 0$, for all $\mathbf{x} \in \mathbb{R}^{n}, \delta>0$ and $i=1, \cdots, n$. A function $f: \mathbb{R}^{n} \rightarrow \mathbb{R}$ is is said to be supermodular, if $\Delta_{i}^{\delta} \Delta_{j}^{\varepsilon} f(\mathbf{x}) \geq 0$, for all $\mathbf{x} \in \mathbb{R}^{n}, \delta, \varepsilon>0$ and $1 \leq i<j \leq n$. Equivalently, a function $f: \mathbb{R}^{n} \rightarrow \mathbb{R}$ is said to be supermodular if for any $\mathbf{x}, \mathbf{y} \in \mathbb{R}^{n}$ it holds that

$$
f(\mathbf{x})+f(\mathbf{y}) \leq f(\mathbf{x} \wedge \mathbf{y})+f(\mathbf{x} \vee \mathbf{y}),
$$

where the operators $\wedge$ and $\vee$ denote coordinatewise minimum and maximum, respectively. A function $f$ is supermodular if and only if $-f$ is submodular. A function $f: \mathbb{R}^{n} \rightarrow \mathbb{R}$ is said to be componentwise convex if $f$ is convex in each argument when the other arguments are hold fixed. A function $f: \mathbb{R}^{n} \rightarrow \mathbb{R}$ is said to be directionally convex, if $\Delta_{i}^{\delta} \Delta_{j}^{\varepsilon} f(\mathbf{x}) \geq 0$, for all $\mathbf{x} \in \mathbb{R}^{n}, \delta, \varepsilon>0$ and $1 \leq i, j \leq n$. That is $f: \mathbb{R}^{n} \rightarrow \mathbb{R}$ is directionally convex if it is supermodular and componentwise convex. Directional convexity neither implies, nor is implied by, conventional convexity. The supermodular order compares only the dependence structure of vectors with fixed equal marginals, whereas the increasing directionally convex order also compares the marginals both invariability and location, where the marginals are possibly different. However, a univariate function is directionally convex if, and only if, it is convex. A function $f: \mathbb{R}^{n} \rightarrow \mathbb{R}$ is said to be $\Delta$-monotone function, if for all $\left\{i_{1}, \cdots, i_{k}\right\} \subset\{1, \cdots, n\}$ and every $\delta_{1}, \cdots, \delta_{k}>0$, $\Delta_{i_{1}}^{\delta_{1}} \cdots \Delta_{i_{k}}^{\delta_{k}} f(\mathbf{x}) \geq 0$, for all $\mathbf{x} \in \mathbb{R}^{n}, \delta, \varepsilon>0$.

Let us now recall the definitions of stochastic orders that will be used later.

Let $\mathcal{F}$ be some class of measurable functions $f: \mathbb{R}^{n} \rightarrow \mathbb{R}$, for two random vectors $\mathbf{X}$ and $\mathbf{Y}$ in $\mathbb{R}^{n}$, we say that $\mathbf{X} \leq_{\mathcal{F}} \mathbf{Y}$ if $E[f(\mathbf{X})] \leq E[f(\mathbf{Y})]$ holds for all $f \in \mathcal{F}$ whenever the expectation is well defined. We list a few important examples as follows.

- Usual stochastic order: $\mathbf{X} \leq_{s t} \mathbf{Y}$, if $E[f(\mathbf{X})] \leq E[f(\mathbf{Y})]$ for all increasing functions $f: \mathbb{R}^{n} \rightarrow \mathbb{R}$.

- Convex order: $\mathbf{X} \leq_{c x} \mathbf{Y}$, if $E[f(\mathbf{X})] \leq E[f(\mathbf{Y})]$ for all convex functions $f: \mathbb{R}^{n} \rightarrow \mathbb{R}$.

- Linear convex order: $\mathbf{X} \leq_{l c x} \mathbf{Y}$, if $E\left[f\left(\mathbf{a}^{\prime} \mathbf{X}\right)\right] \leq E\left[f\left(\mathbf{a}^{\prime} \mathbf{Y}\right)\right]$ for all $\mathbf{a} \in \mathbb{R}^{n}$ and for all convex functions $f: \mathbb{R}^{n} \rightarrow \mathbb{R}$.

- Increasing convex order: $\mathbf{X} \leq_{i c x} \mathbf{Y}$, if $E[f(\mathbf{X})] \leq E[f(\mathbf{Y})]$ for all increasing convex functions $f: \mathbb{R}^{n} \rightarrow \mathbb{R}$. 
- Componentwise convex order: $\mathbf{X} \leq_{c c x} \mathbf{Y}$, if $E[f(\mathbf{X})] \leq E[f(\mathbf{Y})]$ for all componentwise convex functions $f: \mathbb{R}^{n} \rightarrow \mathbb{R}$.

- Increasing componentwise convex order: $\mathbf{X} \leq_{i c c x} \mathbf{Y}$, if $E[f(\mathbf{X})] \leq E[f(\mathbf{Y})]$ for all increasing componentwise convex functions $f: \mathbb{R}^{n} \rightarrow \mathbb{R}$.

- Supermodular order: $\mathbf{X} \leq_{s m} \mathbf{Y}$, if $E[f(\mathbf{X})] \leq E[f(\mathbf{Y})]$ for all supermodular functions $f: \mathbb{R}^{n} \rightarrow \mathbb{R}$.

- Increasing supermodular order: $\mathbf{X} \leq_{i s m} \mathbf{Y}$, if $E[f(\mathbf{X})] \leq E[f(\mathbf{Y})]$ for all increasing supermodular functions $f: \mathbb{R}^{n} \rightarrow \mathbb{R}$.

- Directionally convex order: $\mathbf{X} \leq_{d c x} \mathbf{Y}$, if $E[f(\mathbf{X})] \leq E[f(\mathbf{Y})]$ for all directionally convex functions $f: \mathbb{R}^{n} \rightarrow \mathbb{R}$.

- Increasing directionally convex: $\mathbf{X} \leq_{i d c x} \mathbf{Y}$, if $E[f(\mathbf{X})] \leq E[f(\mathbf{Y})]$ for all increasing directionally convex functions $f: \mathbb{R}^{n} \rightarrow \mathbb{R}$.

The componentwise convex order was introduced in Mosler (1982), the directionally convex was introduced in Shaked and Shanthikumar (1990) and the increasing directionally convex was introduced in Meester and Shanthikumar (1993).

For a random vector $\mathbf{X}=\left(X_{1}, \cdots, X_{n}\right)$, we denote by

$$
F_{\mathbf{X}}(\mathbf{t}):=P(\mathbf{X} \leq \mathbf{t})=P\left(X_{1} \leq t_{1}, \cdots, X_{n} \leq t_{n}\right), \mathbf{t}=\left(t_{1}, \cdots, t_{n}\right) \in \mathbb{R}^{n},
$$

and

$$
\bar{F}_{\mathbf{X}}(\mathbf{t}):=P(\mathbf{X}>\mathbf{t})=P\left(X_{1}>t_{1}, \cdots, X_{n}>t_{n}\right), \mathbf{t}=\left(t_{1}, \cdots, t_{n}\right) \in \mathbb{R}^{n},
$$

the multivariate distribution function and the multivariate survival function, respectively. The following definition is taking from Müller and Scarsini (2000).

Definition 2.4. (a) Assume that $\mathbf{X}, \mathbf{Y} \in \mathbb{R}^{n}$ are two random vectors.

(a) $\mathbf{X}$ is said to be smaller than $\mathbf{Y}$ in the upper orthant order, written $\mathbf{X} \leq_{u o} \mathbf{Y}$, if $\bar{F}_{\mathbf{X}}(\mathbf{t}) \leq \bar{F}_{\mathbf{Y}}(\mathbf{t})$ for all $\mathbf{t} \in \mathbb{R}^{n}$.

(b) $\mathbf{X}$ is said to be smaller than $\mathbf{Y}$ in the upper orthant order, written $\mathbf{X} \leq_{l o} \mathbf{Y}$, if $F_{\mathbf{X}}(\mathbf{t}) \leq F_{\mathbf{Y}}(\mathbf{t})$ for all $\mathbf{t} \in \mathbb{R}^{n}$.

(c) $\mathbf{X}$ is said to be smaller than $\mathbf{Y}$ in the concordance order, written $\mathbf{X} \leq_{c} \mathbf{Y}$, if both $\mathbf{X} \leq_{u o} \mathbf{Y}$ and $\mathbf{X} \leq_{l o} \mathbf{Y}$ hold.

The orthant orders have been treated by Shaked and Shanthikumar (1994) and the concordance order was introduced by Joe (1990). We have the implication $\mathbf{X} \leq_{s m} \mathbf{Y} \Rightarrow \mathbf{X} \leq_{u o} \mathbf{Y}$ and $\mathbf{X} \leq_{l o} \mathbf{Y}$, and hence also $\mathbf{X} \leq_{s m} \mathbf{Y} \Rightarrow \mathbf{X} \leq_{c} \mathbf{Y}$.

The upper orthant order can be defined alternatively by $\Delta$-monotone functions. The following lemma can be founded in Rüschendorf (1980). 
Lemma 2.1. $\mathbf{X} \leq_{u o} \mathbf{Y}$ if and only if $E[f(\mathbf{X})] \leq E[f(\mathbf{Y})]$ holds for all $\Delta$-monotone functions $f: \mathbb{R}^{n} \rightarrow \mathbb{R}$.

The following necessary and sufficient conditions for several important stochastic orders can be found in Denuit and Müller (2002) and Arlotto and Scarsini (2009).

1. $\mathbf{X} \leq_{s m} \mathbf{Y}$ if, and only if $E[f(\mathbf{X})] \leq E[f(\mathbf{Y})]$ holds for all twice differentiable functions $f: \mathbb{R}^{n} \rightarrow \mathbb{R}$ satisfying $\frac{\partial^{2}}{\partial x_{i} \partial x_{j}} f(\mathbf{x}) \geq 0$ for $\mathbf{x} \in \mathbb{R}^{n}$ and all $1 \leq i<j \leq n$.

2. $\mathbf{X} \leq_{\text {ism }} \mathbf{Y}$ if, and only if $E[f(\mathbf{X})] \leq E[f(\mathbf{Y})]$ holds for all twice differentiable functions $f: \mathbb{R}^{n} \rightarrow \mathbb{R}$ satisfying $\frac{\partial}{\partial x_{i}} f(\mathbf{x}) \geq 0$ for $\mathbf{x} \in \mathbb{R}^{n}$ and all $1 \leq i \leq n$, and $\frac{\partial^{2}}{\partial x_{i} \partial x_{j}} f(\mathbf{x}) \geq 0$ for $\mathbf{x} \in \mathbb{R}^{n}$ and all $1 \leq i<j \leq n$.

3. $\mathbf{X} \leq_{d c x} \mathbf{Y}$ if, and only if, $E[f(\mathbf{X})] \leq E[f(\mathbf{Y})]$ holds for all twice differentiable functions $f: \mathbb{R}^{n} \rightarrow \mathbb{R}$ satisfying $\frac{\partial^{2}}{\partial x_{i} \partial x_{j}} f(\mathbf{x}) \geq 0$ for $\mathbf{x} \in \mathbb{R}^{n}$ and all $1 \leq i, j \leq n$.

4. $\mathbf{X} \leq_{i d c x} \mathbf{Y}$ if, and only if $E[f(\mathbf{X})] \leq E[f(\mathbf{Y})]$ holds for all twice differentiable functions $f: \mathbb{R}^{n} \rightarrow \mathbb{R}$ satisfying $\frac{\partial}{\partial x_{i}} f(\mathbf{x}) \geq 0$ for $\mathbf{x} \in \mathbb{R}^{n}$ and all $1 \leq i \leq n$, and $\frac{\partial^{2}}{\partial x_{i} \partial x_{j}} f(\mathbf{x}) \geq 0$ for $\mathbf{x} \in \mathbb{R}^{n}$ and all $1 \leq i, j \leq n$.

5. $\mathbf{X} \leq_{u o} \mathbf{Y}$ if, and only if $E[f(\mathbf{X})] \leq E[f(\mathbf{Y})]$ holds for all infinitely differentiable functions $f: \mathbb{R}^{n} \rightarrow$ $\mathbb{R}$ satisfying $\frac{\partial^{k}}{\partial x_{i_{1}} \cdots \partial x_{i_{k}}} f(\mathbf{x}) \geq 0$ for $\mathbf{x} \in \mathbb{R}^{n}$ and all $1 \leq i_{1}<\cdots \leq i_{k} \leq n$.

6. $\mathbf{X} \leq_{c c x} \mathbf{Y}$ if, and only if $E[f(\mathbf{X})] \leq E[f(\mathbf{Y})]$ holds for all twice differentiable functions $f: \mathbb{R}^{n} \rightarrow \mathbb{R}$ satisfying $\frac{\partial^{2}}{\partial x_{i}^{2}} f(\mathbf{x}) \geq 0$ for $\mathbf{x} \in \mathbb{R}^{n}$ and all $1 \leq i \leq n$.

7. $\mathbf{X} \leq_{i c c x} \mathbf{Y}$ if, and only if $E[f(\mathbf{X})] \leq E[f(\mathbf{Y})]$ holds for all twice differentiable functions $f: \mathbb{R}^{n} \rightarrow \mathbb{R}$ satisfying $\frac{\partial}{\partial x_{i}} f(\mathbf{x}) \geq 0$ and $\frac{\partial^{2}}{\partial x_{i}^{2}} f(\mathbf{x}) \geq 0$ for $\mathbf{x} \in \mathbb{R}^{n}$ and all $1 \leq i \leq n$.

We first list the results of stochastic orderings for univariate elliptical distributions. For the case of univariate normal distributions can be found in Müller (2001).

Lemma 2.2. Let $X \sim E_{1}\left(\mu_{x}, \sigma_{y}^{2}, \phi\right)$ and $Y \sim E_{1}\left(\mu_{y}, \sigma_{y}^{2}, \phi\right)$. Then

(i) $X \leq_{s t} Y$ if and only if $\mu_{x} \leq \mu_{y}$ and $\sigma_{x}=\sigma_{y}$, provided that $X$ and $Y$ supported on $\mathbb{R}$ ( Davidov and Peddada(2013));

(ii) $X \leq_{c x} Y$ if and only if $\mu_{x}=\mu_{y}$ and $\sigma_{x} \leq \sigma_{y}$ (Pan et al. (2016));

(iii) $X \leq_{i c x} Y$ if and only if $\mu_{x} \leq \mu_{y}$ and $\sigma_{x} \leq \sigma_{y}$ (Pan et al. (2016)).

Now we list the results of stochastic orderings for multivariate elliptical distributions.

Lemma 2.3. (Pan et al. (2016)) Let $\mathbf{X} \sim E_{n}\left(\boldsymbol{\mu}_{x}, \boldsymbol{\Sigma}_{x}, \phi\right)$ and $\mathbf{Y} \sim E_{n}\left(\boldsymbol{\mu}_{y}, \boldsymbol{\Sigma}_{y}, \phi\right)$. Then the following statements are equivalent:

(1) $\boldsymbol{\mu}_{x}=\boldsymbol{\mu}_{y}$ and $\boldsymbol{\Sigma}_{y}-\boldsymbol{\Sigma}_{x}$ is positively semi-definite; 
(2) $\mathbf{X} \leq_{l c x} \mathbf{Y}$;

(3) $\mathbf{X} \leq_{c x} \mathbf{Y}$.

For the case of increasing convex order, the sufficient and necessary conditions seem to be unknown. The following sufficient condition for the increasing convex order can be found in Pan et al. (2016). The results for the case of multivariate normal distributions can be found in Müller (2001).

Lemma 2.4. Let $\mathbf{X} \sim E_{n}\left(\boldsymbol{\mu}_{x}, \boldsymbol{\Sigma}_{x}, \phi\right)$ and $\mathbf{Y} \sim E_{n}\left(\boldsymbol{\mu}_{y}, \boldsymbol{\Sigma}_{y}, \phi\right)$.

(i) If $\boldsymbol{\mu}_{x} \leq \boldsymbol{\mu}_{y}$ and $\boldsymbol{\Sigma}_{y}-\boldsymbol{\Sigma}_{x}$ is positively semi-definite, then $\mathbf{X} \leq_{i c x} \mathbf{Y}$.

(ii) If $\mathbf{X} \leq_{i c x} \mathbf{Y}$, then $\boldsymbol{\mu}_{x} \leq \boldsymbol{\mu}_{y}$ and $\mathbf{a}^{\prime}\left(\boldsymbol{\Sigma}_{y}-\boldsymbol{\Sigma}_{x}\right) \mathbf{a} \geq 0$ for all $\mathbf{a} \geq 0$.

\subsection{Some background on the elliptical distributions}

The class of multivariate elliptical distributions is a natural extension to the class of multivariate Normal distributions. We follow the notation of Cambanis et al. (1981) and Fang et al. (1990). An $n \times 1$ random vector $X=\left(X_{1}, X_{2}, \cdots, X_{n}\right)^{\prime}$ is said to have an elliptically symmetric distribution if its characteristic function has the form $e^{i \mathbf{t}^{\prime} \boldsymbol{\mu}} \phi\left(\mathbf{t}^{\prime} \boldsymbol{\Sigma} \mathbf{t}\right)$ for all $\mathbf{t} \in \mathbb{R}^{n}$, denoted $\mathbf{X} \sim E_{n}(\boldsymbol{\mu}, \boldsymbol{\Sigma}, \phi)$, where $\phi \in \Psi_{n}$ is called the characteristic generator satisfying $\phi(0)=1, \boldsymbol{\mu}$ ( $n$-dimensional vector) is its location parameter and $\boldsymbol{\Sigma}(n \times n$ matrix with $\boldsymbol{\Sigma} \succeq \mathbf{O})$ is its dispersion matrix (or scale matrix). The mean vector $E(\mathbf{X})$ (if it exists) coincides with the location vector and the covariance matrix $\operatorname{Cov}(\mathbf{X})$ (if it exists), being $-2 \phi^{\prime}(0) \boldsymbol{\Sigma}$. It is interesting to note that in the one-dimensional case, the class of elliptical distributions consists mainly of the class of symmetric distributions which include well-known distributions like Normal and Student $t$ distributions. It is well known that $\mathbf{X}$ admits the stochastic representation

$$
\mathbf{X}=\boldsymbol{\mu}+R \mathbf{A}^{\prime} \mathbf{U}^{(n)},
$$

where $\mathbf{A}$ is a square matrix such that $\mathbf{A}^{\prime} \mathbf{A}=\boldsymbol{\Sigma}, \mathbf{U}^{(n)}$ is uniformly distributed on the unit sphere $\mathcal{S}^{n-1}=\left\{\mathbf{u} \in \mathbb{R}^{n}: \mathbf{u}^{\prime} \mathbf{u}=1\right\}, R \geq 0$ is the random variable with $R \sim F$ in $[0, \infty)$ called the generating variate and $F$ is called the generating distribution function, $R$ and $\mathbf{U}^{(n)}$ are independent. The mean vector $E(\mathbf{X})$ exists if and only if $E(R)$ exists and $E(\mathbf{X})=\boldsymbol{\mu}$; The covariance matrix $\operatorname{Cov}(\mathbf{X})$ exists if and only if $E\left(R^{2}\right)$ exists and $\operatorname{Cov}(\mathbf{X})=\frac{1}{n} E\left(R^{2}\right) \boldsymbol{\Sigma}$. In general, an elliptically distributed random vector $\mathbf{X} \sim E_{n}(\boldsymbol{\mu}, \boldsymbol{\Sigma}, \phi)$ does not necessarily possess a density. It is well known that $\mathbf{X}$ has a density if and only if $R$ has a density and $\boldsymbol{\Sigma} \succ \mathbf{O}$. The density has the form

$$
f(\mathbf{x})=c_{n}|\boldsymbol{\Sigma}|^{-\frac{1}{2}} g_{n}\left((\mathbf{x}-\boldsymbol{\mu})^{\prime} \boldsymbol{\Sigma}^{-1}(\mathbf{x}-\boldsymbol{\mu})\right), \mathbf{x} \in \mathbb{R}^{n},
$$

for some nonnegative function $g_{n}$ called the density generator and for some constant $c_{n}$ called the normalizing constant. One sometimes writes $X \sim E_{n}\left(\boldsymbol{\mu}, \boldsymbol{\Sigma}, g_{n}\right)$ for the $n$-dimensional elliptical distributions generated from the function $g_{n}$.

The class of elliptical distributions possesses the linearity property. Consider the affine transformations of the form $\mathbf{Y}=\mathbf{B X}+\mathbf{b}$ of a random vector $\mathbf{X} \sim E_{n}(\boldsymbol{\mu}, \boldsymbol{\Sigma}, \phi)$, where $\mathbf{B}$ is a $m \times n$ matrix with $m<n$ 
and $\operatorname{rank}(\mathbf{B})=m$ and $\mathbf{b} \in \mathbb{R}^{m}$. Then $\mathbf{Y} \sim E_{n}\left(\mathbf{B} \boldsymbol{\mu}+\mathbf{b}, \mathbf{B} \mathbf{\Sigma} \mathbf{B}^{\prime}, \phi\right)$. Taking $B=\left(\alpha_{1}, \cdots, \alpha_{n}\right):=\boldsymbol{\alpha}^{\prime}$ leads to

$$
\boldsymbol{\alpha}^{\prime} \mathbf{X} \sim E_{1}\left(\boldsymbol{\alpha}^{\prime} \boldsymbol{\mu}, \boldsymbol{\alpha}^{\prime} \boldsymbol{\Sigma} \boldsymbol{\alpha}, \phi\right)
$$

In particular,

$$
X_{k} \sim \operatorname{Ell}_{1}\left(\mu_{k}, \sigma_{k}^{2}, \phi\right), \quad k=1,2, \cdots, n,
$$

and

$$
\sum_{k=1}^{n} X_{k} \sim \operatorname{Ell}_{1}\left(\sum_{k=1}^{n} \mu_{k}, \sum_{k=1}^{n} \sum_{l=1}^{n} \sigma_{k l}, \phi\right) .
$$

\subsection{An identity for multivariate elliptical distributions}

If $f: \mathbb{R}^{n} \rightarrow \mathbb{R}$ is twice continuously differentiable, we write as usual

$$
\nabla f(\mathbf{x})=\left(\frac{\partial}{\partial x_{1}} f(\mathbf{x}), \cdots, \frac{\partial}{\partial x_{n}} f(\mathbf{x})\right)^{\prime}, \mathbf{H}_{f}(\mathbf{x})=\left(\frac{\partial^{2}}{\partial x_{i} \partial x_{i}} f(\mathbf{x})\right)_{n \times n}
$$

for the gradient and the Hessian matrix of $f$. It is well known that $f$ is convex if and only if $\mathbf{H}_{f}(\mathbf{x})$ is positive semidefinite for any $\mathbf{x} \in \mathbb{R}^{n} ; f$ is strictly convex if and only if $\mathbf{H}_{f}(\mathbf{x})$ is positive definite for any $\mathbf{x} \in \mathbb{R}^{n}$. A function is supermodular if and only if its Hessian has nonnegative off-diagonal elements, i.e. $f$ is supermodular if and only if $\frac{\partial^{2}}{\partial x_{i} \partial x_{i}} f(\mathbf{x}) \geq 0$ for every $i \neq j$ and $\mathbf{x} \in \mathbb{R}^{n}$ (c.f. Carter (2001, Proposition $4.2)$ ).

The following two results can be found in Houdré et al. (1998), Müller (2001) and Denuit and Müller (2002) in the multivariate normal case. Ding and Zhang (2004) extended the result from multivariate normal distributions to Kotz-type distributions which form an important class of elliptically symmetric distributions. We develop an identity for multivariate elliptical distributions which may be of independent interest.

Lemma 2.5. Let $\mathbf{X} \sim \operatorname{Ell}_{n}\left(\boldsymbol{\mu}^{x}, \boldsymbol{\Sigma}^{x}, \psi\right)$ and $\mathbf{Y} \sim \operatorname{Ell}_{n}\left(\boldsymbol{\mu}^{y}, \boldsymbol{\Sigma}^{y}, \psi\right)$, with $\boldsymbol{\Sigma}^{x}$ and $\boldsymbol{\Sigma}^{y}$ positive definite. Let $\phi_{\lambda}$ be the density function of

$$
E l l_{n}\left(\lambda \boldsymbol{\mu}^{y}+(1-\lambda) \boldsymbol{\mu}^{x}, \lambda \boldsymbol{\Sigma}^{y}+(1-\lambda) \boldsymbol{\Sigma}^{x}, \psi\right), 0 \leq \lambda \leq 1,
$$

and $\phi_{1 \lambda}$ be the density function of

$$
E l l_{n}\left(\lambda \boldsymbol{\mu}^{y}+(1-\lambda) \boldsymbol{\mu}^{x}, \lambda \boldsymbol{\Sigma}^{y}+(1-\lambda) \boldsymbol{\Sigma}^{x}, \psi_{1}\right), 0 \leq \lambda \leq 1,
$$

where

$$
\psi_{1}(u)=\frac{1}{E\left(R^{2}\right)} \int_{0}^{\infty}{ }_{0} F_{1}\left(\frac{n}{2}+1 ;-\frac{r^{2} u}{4}\right) r^{2} P(R \in d r) .
$$

Here

$$
{ }_{0} F_{1}(\gamma ; z)=\sum_{k=0}^{\infty} \frac{1}{(\gamma)_{k}} \frac{z^{k}}{k !},
$$

is the generalized hypergeometric series of order $(0,1), R$ is defined by (2.1) with $E\left(R^{2}\right)<\infty$. Moreover, assume that $f: \mathbb{R}^{n} \rightarrow \mathbb{R}$ is twice continuously differentiable and satisfies some polynomial growth conditions at infinity:

$$
f(\mathbf{x})=O(\|\mathbf{x}\|), \nabla f(\mathbf{x})=O(\|\mathbf{x}\|)
$$


Then

$$
\begin{aligned}
E[f(\mathbf{Y})]-E[f(\mathbf{X})]= & \int_{0}^{1} \int_{\mathbb{R}^{n}}\left(\boldsymbol{\mu}^{y}-\boldsymbol{\mu}^{x}\right)^{\prime} \nabla f(\mathbf{x}) \phi_{\lambda}(\mathbf{x}) d \mathbf{x} d \lambda \\
& +\frac{E\left(R^{2}\right)}{2 n} \int_{0}^{1} \int_{\mathbb{R}^{n}} \operatorname{tr}\left\{\left(\boldsymbol{\Sigma}^{y}-\boldsymbol{\Sigma}^{x}\right) \mathbf{H}_{f}(\mathbf{x})\right\} \phi_{1 \lambda}(\mathbf{x}) d \mathbf{x} d \lambda
\end{aligned}
$$

where $\operatorname{tr}(A)$ denotes the trace of the matrix $A$.

Proof See Appendix 2.

Using Lemma 2.5 and the same argument as in the proof of Corollary 3 in Müller (2001) we have

Corollary 2.1. Let $\mathbf{X} \sim \operatorname{Ell}_{n}\left(\boldsymbol{\mu}^{x}, \boldsymbol{\Sigma}^{x}, \psi\right)$ and $\mathbf{Y} \sim \operatorname{Ell}_{n}\left(\boldsymbol{\mu}^{y}, \boldsymbol{\Sigma}^{y}, \psi\right)$, with $\boldsymbol{\Sigma}^{x}$ and $\boldsymbol{\Sigma}^{y}$ positive definite or positive semidefinite, and assume that $f: \mathbb{R}^{n} \rightarrow \mathbb{R}$ satisfies the conditions of Lemma 2.5. Then $E[f(\mathbf{X})] \leq E[f(\mathbf{Y})]$ if the following two conditions hold for all $\mathbf{x} \in \mathbb{R}^{n}$ :

$$
\sum_{i=1}^{n}\left(\mu_{i}^{y}-\mu_{i}^{x}\right) \frac{\partial}{\partial x_{i}} f(\mathbf{x}) \geq 0,
$$

and

$$
\sum_{i=1}^{n} \sum_{j=1}^{n}\left(\sigma_{i j}^{y}-\sigma_{i j}^{x}\right) \frac{\partial^{2}}{\partial x_{i} \partial x_{j}} f(\mathbf{x}) \geq 0
$$

\section{Main results}

The following results can be extracted from Davidov and Peddada (2013). The multivariate normal case can be found in Müller (2001). Here we provide a different proof.

Theorem 3.1. Let $\mathbf{X} \sim E_{n}\left(\boldsymbol{\mu}^{x}, \boldsymbol{\Sigma}^{x}, \phi\right)$ and $\mathbf{Y} \sim E_{n}\left(\boldsymbol{\mu}^{y}, \boldsymbol{\Sigma}^{y}, \phi\right)$ be two n-dimensional elliptically distributed random vectors supported on $\mathbb{R}^{n}$. Then $\mathbf{X} \leq_{\text {st }} \mathbf{Y}$ if and only if $\boldsymbol{\mu}^{x} \leq \boldsymbol{\mu}^{y}$ and $\boldsymbol{\Sigma}^{y}=\boldsymbol{\Sigma}^{x}$.

Proof For any increasing twice differential function $f: \mathbb{R}^{n} \rightarrow \mathbb{R}$, the "if" part follows immediately from Corollary 2.1, since $\boldsymbol{\mu}^{x} \leq \boldsymbol{\mu}^{y}, \boldsymbol{\Sigma}^{y}=\boldsymbol{\Sigma}^{x}$ and $\nabla f(\mathbf{x}) \geq 0$ for all $\mathbf{x} \in \mathbb{R}^{n}$ imply that $E[f(\mathbf{Y})] \geq$ $E[f(\mathbf{X})]$. To prove the "only if" part, we choose $f$ to have the forms of $f(\mathbf{x})=h_{1}\left(x_{i}\right)$ and $f(\mathbf{x})=$ $h_{2}\left(x_{i}+x_{j}\right)$, where $h_{1}$ and $h_{2}$ are any two univariate increasing functions, it follows from $\mathbf{X} \leq_{s t} \mathbf{Y}$ that $X_{i} \leq_{s t} Y_{i}$ and $X_{i}+Y_{i} \leq_{s t} X_{j}+Y_{j}$. Note that $\mathbf{X} \sim E_{n}\left(\boldsymbol{\mu}^{x}, \boldsymbol{\Sigma}^{x}, \phi\right)$ and $\mathbf{Y} \sim E_{n}\left(\boldsymbol{\mu}^{y}, \boldsymbol{\Sigma}^{y}, \phi\right)$ lead to $X_{i} \sim E_{1}\left(\mu_{i}^{x}, \sigma_{i i}^{x}, \phi\right)$ and $X_{i}+Y_{i} \sim E_{1}\left(\mu_{i}^{x}+\mu_{j}^{x}, \sigma_{i i}^{x}+\sigma_{j j}^{x}+2 \sigma_{i j}^{x}, \phi\right)$. By the symmetry of elliptical distributions, all $X_{i}, X_{j}, Y_{i}, Y_{j}, X_{i}+Y_{i}$ and $X_{j}+Y_{j}$ are supported on $\mathbb{R}$. Applying Lemma 2.2 (i) we find that $\mu_{i}^{x} \leq \mu_{i}^{y}$ and $\sigma_{i j}^{x}=\sigma_{i j}^{y}$ for all $1 \leq i, j \leq n$. Hence, $\boldsymbol{\mu}^{x} \leq \boldsymbol{\mu}^{y}$ and $\boldsymbol{\Sigma}^{y}=\boldsymbol{\Sigma}^{x}$. This proves the "only if" part.

The following result, due to Pan et al. (2016), generalizes Theorem 4 in Scarsini (1998) and Theorem 6 in Müller (2001) for the multivariate normal case. Here we provided a different proof. 
Theorem 3.2. Let $\mathbf{X} \sim E_{n}\left(\boldsymbol{\mu}^{x}, \boldsymbol{\Sigma}^{x}, \phi\right)$ and $\mathbf{Y} \sim E_{n}\left(\boldsymbol{\mu}^{y}, \boldsymbol{\Sigma}^{y}, \phi\right)$. Then the following statements are equivalent:

(1) $\boldsymbol{\mu}^{y}=\boldsymbol{\mu}^{x}$ and $\boldsymbol{\Sigma}^{y}-\boldsymbol{\Sigma}^{x} \succeq \mathbf{O}$;

(2) $\mathbf{X} \leq_{c x} \mathbf{Y}$;

(3) $\mathbf{X} \leq_{l c x} \mathbf{Y}$.

Proof $(1) \Rightarrow(2)$. For any twice differential convex function $f: \mathbb{R}^{n} \rightarrow \mathbb{R}$, using Lemma 2.5 we get $E[f(\mathbf{Y})] \geq E[f(\mathbf{X})]$ since $f$ is convex if and only if its Hessian matrix $H_{f}$ is positive semi-definite. (2) $\Rightarrow$ (3) is obvious. $(3) \Rightarrow(1)$ is the same as the proof of Theorem 4.1 in Pan et al. (2016).

The following result, due to Pan et al. (2016), generalizes Theorem 7 in Müller (2001) for the multivariate normal case. Here we provided a different proof.

Theorem 3.3. Let $\mathbf{X} \sim E_{n}\left(\boldsymbol{\mu}^{x}, \boldsymbol{\Sigma}^{x}, \phi\right)$ and $\mathbf{Y} \sim E_{n}\left(\boldsymbol{\mu}^{y}, \boldsymbol{\Sigma}^{y}, \phi\right)$. Then the following statements hold:

(1) If $\boldsymbol{\mu}^{x} \leq \boldsymbol{\mu}^{y}$ and $\boldsymbol{\Sigma}^{y}-\boldsymbol{\Sigma}^{x} \succeq \mathbf{O}$, then $\mathbf{X} \leq_{i c x} \mathbf{Y}$.

(2) If $\mathbf{X} \leq_{i c x} \mathbf{Y}$, then $\boldsymbol{\mu}^{y} \geq \boldsymbol{\mu}^{x}$ and $\boldsymbol{\Sigma}^{y}-\boldsymbol{\Sigma}^{x}$ is copositive, i.e., $\mathbf{a}^{\prime}\left(\boldsymbol{\Sigma}^{y}-\boldsymbol{\Sigma}^{x}\right) \mathbf{a} \geq 0$ for all $\mathbf{a} \geq 0$.

Proof (1). For any twice differential increasing convex function $f: \mathbb{R}^{n} \rightarrow \mathbb{R}$, using Lemma 2.5, together with the conditions $\boldsymbol{\mu}^{y} \geq \boldsymbol{\mu}^{x}$ and $\boldsymbol{\Sigma}^{y}-\boldsymbol{\Sigma}^{x} \succeq \mathbf{O}$, we get $E[f(\mathbf{Y})] \geq E[f(\mathbf{X})]$, and thus we have $\mathbf{X} \leq_{i c x} \mathbf{Y}$. The proof of (2) is the same as the proof of Theorem 4.6(2) in Pan et al. (2016).

Remark 3.1. For the case of increasing convex order, there are no sufficient and necessary conditions in the literature even for normal distributions, see Müller (2001) and Pan et al. (2016). We remark that if $\left(\boldsymbol{\Sigma}^{y}-\boldsymbol{\Sigma}^{x}\right) \mathbf{z}=0$ has a positive solution, then $\mathbf{a}^{\prime}\left(\boldsymbol{\Sigma}^{y}-\boldsymbol{\Sigma}^{x}\right) \mathbf{a} \geq 0$ for all $\mathbf{a} \geq 0$ if and only if $\boldsymbol{\Sigma}^{y}-\boldsymbol{\Sigma}^{x} \succeq \mathbf{O}$ (see Theorem A1). So we get the following if-and-only-if characterization of increasing convex order: Assume that $\mathbf{X} \sim E_{n}\left(\boldsymbol{\mu}^{x}, \boldsymbol{\Sigma}^{x}, \phi\right), \mathbf{Y} \sim E_{n}\left(\boldsymbol{\mu}^{y}, \boldsymbol{\Sigma}^{y}, \phi\right)$ and $\left(\boldsymbol{\Sigma}^{y}-\boldsymbol{\Sigma}^{x}\right) \mathbf{z}=0$ has a positive solution. Then $\mathbf{X} \leq_{i c x} \mathbf{Y}$ if and only if $\boldsymbol{\mu}^{y} \geq \boldsymbol{\mu}^{x}$ and $\boldsymbol{\Sigma}^{y}-\boldsymbol{\Sigma}^{x} \succeq \mathbf{O}$.

Remark 3.2. It is easy to see that $\boldsymbol{\Sigma}^{y}-\boldsymbol{\Sigma}^{x} \succeq \mathbf{O}$ implies $\boldsymbol{\Sigma}^{y}-\boldsymbol{\Sigma}^{x}$ is copositive, but conversely is not true. We give an example. Let

$$
\boldsymbol{\Sigma}^{x}=\left(\begin{array}{cc}
\sigma^{2} & \rho_{x} \sigma^{2} \\
\rho_{x} \sigma^{2} & \sigma^{2}
\end{array}\right), \boldsymbol{\Sigma}^{y}=\left(\begin{array}{cc}
\sigma^{2} & \rho_{y} \sigma^{2} \\
\rho_{y} \sigma^{2} & \sigma^{2}
\end{array}\right),
$$

where $\sigma^{2}>0$ and $-1 \leq \rho_{x}<\rho_{y} \leq 1$. Then for all $\mathbf{a}=\left(a_{1}, a_{2}\right)^{\prime} \geq 0, \mathbf{a}^{\prime}\left(\boldsymbol{\Sigma}^{y}-\boldsymbol{\Sigma}^{x}\right) \mathbf{a}=a_{1} a_{2} \sigma^{2}\left(\rho_{y}-\rho_{x}\right) \geq 0$. But for $\mathbf{Z}=\left(z_{1},-z_{1}\right)^{\prime} \in \mathbb{R}^{2}, \mathbf{Z}^{\prime}\left(\boldsymbol{\Sigma}^{y}-\boldsymbol{\Sigma}^{x}\right) \mathbf{Z}=-z_{1}^{2} \sigma^{2}\left(\rho_{y}-\rho_{x}\right) \leq 0$.

The following result generalizes Theorem 11 in Müller (2001) in which the multivariate normal case was considered. 
Theorem 3.4. Let $\mathbf{X} \sim E_{n}\left(\boldsymbol{\mu}^{x}, \boldsymbol{\Sigma}^{x}, \phi\right)$ and $\mathbf{Y} \sim E_{n}\left(\boldsymbol{\mu}^{y}, \boldsymbol{\Sigma}^{y}, \phi\right)$. Then the following statements are equivalent:

(1) $\mathbf{X} \leq_{s m} \mathbf{Y}$.

(2) $\mathbf{X}$ and $\mathbf{Y}$ have the same marginal and $\sigma_{i j}^{x} \leq \sigma_{i j}^{y}$ for all $1 \leq i<j \leq n$.

Proof $(1) \Rightarrow(2)$. If $\mathbf{X} \leq_{s m} \mathbf{Y}$, then $\mathbf{X}$ and $\mathbf{Y}$ necessarily belong to the same Fréchet space. In particular, $\mathbf{X}$ and $\mathbf{Y}$ have the same marginal (see e.g. Müller (2000)). Since the function $f(\mathbf{x})=x_{i} x_{j}$ is supermodular for all $1 \leq i<j \leq n$, we see that $\mathbf{X} \leq_{s m} \mathbf{Y}$ implies $\sigma_{i j}^{x} \leq \sigma_{i j}^{y}$ for all $1 \leq i<j \leq n$.

Since $\mathbf{X} \leq_{s m} \mathbf{Y}$ if and only if $E[f(\mathbf{X})] \leq E[f(\mathbf{Y})]$ holds for all twice differentiable functions $f: \mathbb{R}^{n} \rightarrow \mathbb{R}$ satisfying $\frac{\partial^{2}}{\partial x_{i} \partial x_{j}} f(\mathbf{x}) \geq 0$ for $\mathbf{x} \in \mathbb{R}^{n}$ and all $1 \leq i<j \leq n$, the implication (2) $\Rightarrow(1)$ follows from Lemma 2.5 .

Theorem 3.5. Let $\mathbf{X} \sim E_{n}\left(\boldsymbol{\mu}^{x}, \boldsymbol{\Sigma}^{x}, \phi\right)$ and $\mathbf{Y} \sim E_{n}\left(\boldsymbol{\mu}^{y}, \boldsymbol{\Sigma}^{y}, \phi\right)$ be two n-dimensional elliptically distributed random vectors supported on $\mathbb{R}^{n}$.

(1) If $\boldsymbol{\mu}^{x} \leq \boldsymbol{\mu}^{y}, \sigma_{i i}^{x}=\sigma_{i i}^{y}$ for $i=1,2, \cdots, n$ and $\sigma_{i j}^{x} \leq \sigma_{i j}^{y}$ for all $1 \leq i<j \leq n$, then $\mathbf{X} \leq i s m \mathbf{Y}$.

(2) If $\mathbf{X} \leq_{i s m} \mathbf{Y}$, then $\boldsymbol{\mu}^{x} \leq \boldsymbol{\mu}^{y}, \sigma_{i i}^{x}=\sigma_{i i}^{y}$ for $i=1,2, \cdots, n$ and $E\left(X_{i} X_{j}\right) \leq E\left(Y_{i} Y_{j}\right)$ for all $1 \leq i<j \leq n$.

Proof (1). For any twice differentiable functions $f: \mathbb{R}^{n} \rightarrow \mathbb{R}$ satisfying $\frac{\partial}{\partial x_{i}} f(\mathbf{x}) \geq 0$ for $\mathbf{x} \in \mathbb{R}^{n}$ and all $1 \leq i \leq n$ and $\frac{\partial^{2}}{\partial x_{i} \partial x_{j}} f(\mathbf{x}) \geq 0$ for $\mathbf{x} \in \mathbb{R}^{n}$ and all $1 \leq i<j \leq n$, using Corollary 2.1, together with the conditions $\boldsymbol{\mu}^{y} \geq \boldsymbol{\mu}^{x}, \sigma_{i i}^{x}=\sigma_{i i}^{y}$ for $i=1,2, \cdots, n$ and $\sigma_{i j}^{x} \leq \sigma_{i j}^{y}$ for all $1 \leq i<j \leq n$, we get $E[f(\mathbf{Y})] \geq E[f(\mathbf{X})]$. Thus, we have $\mathbf{X} \leq_{i s m} \mathbf{Y}$.

(2). $\mathbf{X} \leq_{i s m} \mathbf{Y}$ implies $X_{i} \leq_{s t} Y_{i}$ (Müller and Stoyan (2002. P.114). Applying Lemma 2.1 (i) we find that $\mu_{i}^{x} \leq \mu_{i}^{y}$ and $\sigma_{i j}^{x}=\sigma_{i j}^{y}$ for all $1 \leq i, j \leq n$. Choosing a supermodular $f(\mathbf{x})=x_{i} x_{j}(i \neq j)$, it follows from $\mathbf{X} \leq_{i s m} \mathbf{Y}$ that $E\left(X_{i} X_{j}\right) \leq E\left(Y_{i} Y_{j}\right)$.

Corollary 3.1. Let $\mathbf{X} \sim E_{n}\left(\mathbf{0}, \boldsymbol{\Sigma}^{x}, \phi\right)$ and $\mathbf{Y} \sim E_{n}\left(\mathbf{0}, \boldsymbol{\Sigma}^{y}, \phi\right)$ be two n-dimensional elliptically distributed random vectors supported on $\mathbb{R}^{n}$. Then the following statements are equivalent:

(1) $\mathbf{X} \leq_{i s m} \mathbf{Y}$.

(2) $\sigma_{i i}^{x}=\sigma_{i i}^{y}$ for $i=1,2, \cdots, n$ and $\sigma_{i j}^{x} \leq \sigma_{i j}^{y}$ for all $1 \leq i<j \leq n$.

The following result generalizes Theorem 12 in Müller (2001) in which the multivariate normal case was considered.

Theorem 3.6. Let $\mathbf{X} \sim E_{n}\left(\boldsymbol{\mu}^{x}, \boldsymbol{\Sigma}^{x}, \phi\right)$ and $\mathbf{Y} \sim E_{n}\left(\boldsymbol{\mu}^{y}, \boldsymbol{\Sigma}^{y}, \phi\right)$. Then the following statements are equivalent:

(1) $\mathbf{X} \leq_{d c x} \mathbf{Y}$.

(2) $\boldsymbol{\mu}^{x}=\boldsymbol{\mu}^{y}$ and $\sigma_{i j}^{x} \leq \sigma_{i j}^{y}$ for all $1 \leq i, j \leq n$. 
Proof $(1) \Rightarrow(2)$. Note that the functions $f(\mathbf{x})=x_{i},-x_{i}, x_{i} x_{j}$ are directionally convex for all $1 \leq$ $i, j \leq n$, thus $\boldsymbol{\mu}^{x}=\boldsymbol{\mu}^{y}$ and $\sigma_{i j}^{x} \leq \sigma_{i j}^{y}$ for all $1 \leq i, j \leq n$.

$(2) \Rightarrow(1)$. Since $\mathbf{X} \leq_{d c x} \mathbf{Y}$ if and only if $E[f(\mathbf{X})] \leq E[f(\mathbf{Y})]$ holds for all twice differentiable functions $f: \mathbb{R}^{n} \rightarrow \mathbb{R}$ satisfying $\frac{\partial^{2}}{\partial x_{i} \partial x_{j}} f(\mathbf{x}) \geq 0$ for $\mathbf{x} \in \mathbb{R}^{n}$ and all $1 \leq i, j \leq n$, the implication (2) $\Rightarrow(1)$ follows from Lemma 2.5 .

For increasing directionally convex orders we have

Theorem 3.7. Let $\mathbf{X} \sim E_{n}\left(\boldsymbol{\mu}^{x}, \boldsymbol{\Sigma}^{x}, \phi\right)$ and $\mathbf{Y} \sim E_{n}\left(\boldsymbol{\mu}^{y}, \boldsymbol{\Sigma}^{y}, \phi\right)$. Then the following statements are equivalent:

(1) $\mathbf{X} \leq_{i d c x} \mathbf{Y}$.

(2) $\boldsymbol{\mu}^{x} \leq \boldsymbol{\mu}^{y}$ and $\sigma_{i j}^{x} \leq \sigma_{i j}^{y}$ for all $1 \leq i, j \leq n$.

Proof $(1) \Rightarrow(2)$. Note that the functions $f(\mathbf{x})=x_{i}, x_{i} x_{j}$ are directionally convex for all $1 \leq i, j \leq n$. Thus, $\boldsymbol{\mu}^{x} \leq \boldsymbol{\mu}^{y}$ and $\sigma_{i j}^{x} \leq \sigma_{i j}^{y}$ for all $1 \leq i, j \leq n$.

$(2) \Rightarrow(1)$. Since $\mathbf{X} \leq_{i d c x} \mathbf{Y}$ if and only if $E[f(\mathbf{X})] \leq E[f(\mathbf{Y})]$ holds for all twice differentiable increasing functions $f: \mathbb{R}^{n} \rightarrow \mathbb{R}$ satisfying $\frac{\partial^{2}}{\partial x_{i} \partial x_{j}} f(\mathbf{x}) \geq 0$ for $\mathbf{x} \in \mathbb{R}^{n}$ and all $1 \leq i, j \leq n$, the implication $(2) \Rightarrow(1)$ follows from Lemma 2.5 .

As pointed out by Müller (2001), the if-and-only-if characterization of the upper orthant order for multinormal distributions is not found. The following result generalizes and strengthen Theorem 10 in Müller (2001) in which the multivariate normal case was considered and Theorem 2 in Landsman and Tsanakas (2006) in which the bivariate elliptical distributions was considered.

Theorem 3.8. Let $\mathbf{X} \sim E_{n}\left(\boldsymbol{\mu}^{x}, \boldsymbol{\Sigma}^{x}, \phi\right)$ and $\mathbf{Y} \sim E_{n}\left(\boldsymbol{\mu}^{y}, \boldsymbol{\Sigma}^{y}, \phi\right)$ be two $n$-dimensional elliptically distributed random vectors supported on $\mathbb{R}^{n}$.

(1) If $\boldsymbol{\mu}^{x} \leq \boldsymbol{\mu}^{y}, \sigma_{i i}^{x}=\sigma_{i i}^{y}$ for $i=1,2, \cdots, n$ and $\sigma_{i j}^{x} \leq \sigma_{i j}^{y}$ for all $1 \leq i<j \leq n$, then $\mathbf{X} \leq_{u_{o}} \mathbf{Y}$.

(2) If $\mathbf{X} \leq{ }_{u o} \mathbf{Y}$, then $\boldsymbol{\mu}^{x} \leq \boldsymbol{\mu}^{y}, \sigma_{i i}^{x}=\sigma_{i i}^{y}$ for $i=1,2, \cdots, n$ and $E\left(X_{i} X_{j}\right) \leq E\left(Y_{i} Y_{j}\right)$ for all $1 \leq i<j \leq n$.

Proof (1). For any $\Delta$-monotone functions $f: \mathbb{R}^{n} \rightarrow \mathbb{R}$, using Lemma 2.5, together with the conditions $\boldsymbol{\mu}^{y} \geq \boldsymbol{\mu}^{x}, \sigma_{i i}^{x}=\sigma_{i i}^{y}$ for $i=1,2, \cdots, n$ and $\sigma_{i j}^{x} \leq \sigma_{i j}^{y}$ for all $1 \leq i<j \leq n$, we get $E[f(\mathbf{Y})] \geq E[f(\mathbf{X})]$, and thus we have $\mathbf{X} \leq_{u o} \mathbf{Y}$.

(2). Using the fact that $\mathbf{X} \leq_{u o} \mathbf{Y}$ implies $X_{i} \leq_{s t} Y_{i}$ for all $1 \leq i \leq n$ and Lemma 2.2(i) we get $\boldsymbol{\mu}^{x} \leq \boldsymbol{\mu}^{y}$ and $\sigma_{i i}^{x}=\sigma_{i i}^{y}$ for $i=1,2, \cdots, n$. Choosing $f(\mathbf{x})=x_{i} x_{j}(i \neq j)$, which is a $\Delta$-monotone function, it follows from $\mathbf{X} \leq_{u o} \mathbf{Y}$ that $E\left(X_{i} X_{j}\right) \leq E\left(Y_{i} Y_{j}\right)$. 
Corollary 3.2. Let $\mathbf{X} \sim E_{n}\left(\mathbf{0}, \boldsymbol{\Sigma}^{x}, \phi\right)$ and $\mathbf{Y} \sim E_{n}\left(\mathbf{0}, \boldsymbol{\Sigma}^{y}, \phi\right)$ be two $n$-dimensional elliptically distributed random vectors supported on $\mathbb{R}^{n}$. Then the following statements are equivalent:

(1) $\mathbf{X} \leq_{u o} \mathbf{Y}$.

(2) $\sigma_{i i}^{x}=\sigma_{i i}^{y}$ for $i=1,2, \cdots, n$ and $\sigma_{i j}^{x} \leq \sigma_{i j}^{y}$ for all $1 \leq i<j \leq n$.

The following theorem considers the componentwise convex order. The multivariate normal case can be found in Müller and Stoyan (2002), see also Arlotto and Scarsini (2009).

Theorem 3.9. Let $\mathbf{X} \sim E_{n}\left(\boldsymbol{\mu}^{x}, \boldsymbol{\Sigma}^{x}, \phi\right)$ and $\mathbf{Y} \sim E_{n}\left(\boldsymbol{\mu}^{y}, \boldsymbol{\Sigma}^{y}, \phi\right)$. Then the following statements are equivalent:

(1) $\mathbf{X} \leq_{c c x} \mathbf{Y}$.

(2) $\boldsymbol{\mu}^{x}=\boldsymbol{\mu}^{y}$ and $\sigma_{i i}^{x} \leq \sigma_{i i}^{y}$ for all $1 \leq i \leq n$, and $\sigma_{i j}^{x}=\sigma_{i j}^{y}$ for all $1 \leq i<j \leq n$.

Proof $(1) \Rightarrow(2)$. Note that the functions $f(\mathbf{x})=x_{i},-x_{i}, x_{i}^{2}, x_{i} x_{j},-x_{i} x_{j}$ are componentwise convex for all $1 \leq i, j \leq n$. Thus, we get $\boldsymbol{\mu}^{x}=\boldsymbol{\mu}^{y}, \sigma_{i i}^{x} \leq \sigma_{i i}^{y}$ for all $1 \leq i \leq n$ and $\sigma_{i j}^{x}=\sigma_{i j}^{y}$ for all $1 \leq i<j \leq n$.

$(2) \Rightarrow(1)$. For any twice differentiable functions $f: \mathbb{R}^{n} \rightarrow \mathbb{R}$ satisfying $\frac{\partial^{2}}{\partial x_{i}^{2}} f(\mathbf{x}) \geq 0$ for $\mathbf{x} \in \mathbb{R}^{n}$ and all $1 \leq i \leq n$, using Lemma 2.5, together with the conditions $\boldsymbol{\mu}^{x}=\boldsymbol{\mu}^{y}, \sigma_{i i}^{x} \leq \sigma_{i i}^{y}$ for all $1 \leq i \leq n$ and $\sigma_{i j}^{x}=\sigma_{i j}^{y}$ for all $1 \leq i<j \leq n$, we get $E[f(\mathbf{X})] \leq E[f(\mathbf{Y})]$. Thus $\mathbf{X} \leq_{c c x} \mathbf{Y}$.

Similarly, we establish the result for increasing componentwise convex order as follows.

Theorem 3.10. Let $\mathbf{X} \sim E_{n}\left(\boldsymbol{\mu}^{x}, \boldsymbol{\Sigma}^{x}, \phi\right)$ and $\mathbf{Y} \sim E_{n}\left(\boldsymbol{\mu}^{y}, \boldsymbol{\Sigma}^{y}, \phi\right)$. Then the following statements are equivalent:

(1) $\mathbf{X} \leq_{i c c x} \mathbf{Y}$.

(2) $\mu_{i}^{x} \leq \mu_{i}^{y}$ and $\sigma_{i i}^{x} \leq \sigma_{i i}^{y}$ for all $1 \leq i \leq n$, and $\sigma_{i j}^{x}=\sigma_{i j}^{y}$ for all $1 \leq i<j \leq n$.

At the end of this section, we will consider the copositive and completely positive orders for multivariate elliptical random variables. The multivariate normal case can be found in Pan et al. (2016) pointed out that it is still unknown whether such a characterization holds for multivariate elliptical distributions. Before we state Theorem 3.11, we first give the following definitions.

Definition 3.1 (Arlotto and Scarsini (2009)) An $n \times n$ matrix A is called copositive if the quadratic form $\mathbf{x}^{\prime} \mathbf{A} \mathbf{x} \geq 0$ for all $\mathbf{x} \geq 0$, and $\mathbf{A}$ is called completely positive if there exists a nonnegative $m \times n$ matrix $\mathbf{B}$ such that $\mathbf{A}=\mathbf{B}^{\prime} \mathbf{B}$.

Denote by $\mathcal{C}_{c o p}$ the cone of copositive matrices and as $\mathcal{C}_{c p}$ the cone of completely positive matrices. Let $\mathcal{C}_{c o p}^{*}$ and $\mathcal{C}_{c p}^{*}$ be the dual of $\mathcal{C}_{c o p}$ and $\mathcal{C}_{c p}$, respectively. It is well known that (see Arlotto and Scarsini $(2009)) \mathcal{C}_{c o p}^{*}=\mathcal{C}_{c p}$ and $\mathcal{C}_{c p}^{*}=\mathcal{C}_{c o p}$. 
The following Hessian orders can be defined (see Arlotto and Scarsini (2009)).

(a) $X \leq_{c p} Y$ if $E[f(\mathbf{X})] \leq E[f(\mathbf{Y})]$ holds for all functions $f$ such that $\mathbf{H}_{f}(\mathbf{x}) \in \mathcal{C}_{c p}$.

(b) $X \leq_{\text {cop }} Y$ if $E[f(\mathbf{X})] \leq E[f(\mathbf{Y})]$ holds for all functions $f$ such that $\mathbf{H}_{f}(\mathbf{x}) \in \mathcal{C}_{\text {cop }}$.

Theorem 3.11. Let $\mathbf{X} \sim E_{n}\left(\boldsymbol{\mu}^{x}, \boldsymbol{\Sigma}^{x}, \phi\right)$ and $\mathbf{Y} \sim E_{n}\left(\boldsymbol{\mu}^{y}, \boldsymbol{\Sigma}^{y}, \phi\right)$. Then

(1) $\mathbf{X} \leq_{c p} \mathbf{Y}$ if and only if $\boldsymbol{\mu}^{x}=\boldsymbol{\mu}^{y}$ and $\boldsymbol{\Sigma}^{y}-\boldsymbol{\Sigma}^{x}$ is copositive.

(2) $\mathbf{X} \leq_{c o p} \mathbf{Y}$ if and only if $\boldsymbol{\mu}^{x}=\boldsymbol{\mu}^{y}$ and $\boldsymbol{\Sigma}^{y}-\boldsymbol{\Sigma}^{x}$ is completely copositive.

Proof We prove (1) and the proof of (2) is similar. "If part": Consider the functions $f_{i}(\mathbf{x})=x_{i},-x_{i}$ $(1 \leq i \leq n)$. Observe that $\mathbf{H}_{f_{i}}(\mathbf{x})=\mathbf{O} \in \mathcal{C}_{c p}$. Thus, $\mathbf{X} \leq_{c p} \mathbf{Y}$ implies $\boldsymbol{\mu}^{x}=\boldsymbol{\mu}^{y}$. Let $E(\mathbf{X})=E(\mathbf{Y})=\boldsymbol{\mu}$. For any symmetric $n \times n$ matrix $\mathbf{A} \in \mathcal{C}_{c p}$, define a function $f$ as

$$
f(\mathbf{x})=\frac{1}{2}(\mathbf{x}-\boldsymbol{\mu})^{\prime} \mathbf{A}(\mathbf{x}-\boldsymbol{\mu}) .
$$

Observe that $\mathbf{H}_{f}(\mathbf{x})=\mathbf{A}$ for all $\mathbf{x}$, and thus $\mathbf{X} \leq_{c p} \mathbf{Y}$ implies $E[f(\mathbf{X})] \leq E[f(\mathbf{Y})]$, which is equivalent to

$$
E(\mathbf{X}-\boldsymbol{\mu})^{\prime} \mathbf{A}(\mathbf{X}-\boldsymbol{\mu}) \leq E(\mathbf{Y}-\boldsymbol{\mu})^{\prime} \mathbf{A}(\mathbf{Y}-\boldsymbol{\mu}) .
$$

It follows from the above that $-2 \phi^{\prime}(0) \operatorname{tr}\left(\boldsymbol{\Sigma}^{x} \mathbf{A}\right) \leq-2 \phi^{\prime}(0) \operatorname{tr}\left(\boldsymbol{\Sigma}^{y} \mathbf{A}\right)$. Therefore, $\operatorname{tr}\left(\left(\boldsymbol{\Sigma}^{y}-\boldsymbol{\Sigma}^{x}\right) \mathbf{A}\right) \geq 0$. Since $\mathbf{A} \in \mathcal{C}_{c p}$ is arbitrary, we conclude that $\boldsymbol{\Sigma}^{y}-\boldsymbol{\Sigma}^{x} \in \mathcal{C}_{c p}^{*}$. Hence, $\boldsymbol{\Sigma}^{y}-\boldsymbol{\Sigma}^{x}$ is copositive, since $\mathcal{C}_{c p}^{*}=\mathcal{C}_{c o p}$. "Only if part": For any $f$ such that $\mathbf{H}_{f}(\mathbf{x}) \in \mathcal{C}_{c p}$, using Lemma 2.5, together with the condition $\boldsymbol{\mu}^{x}=\boldsymbol{\mu}^{y}$ and the fact that $\boldsymbol{\Sigma}^{y}-\boldsymbol{\Sigma}^{x}$ is copositive, yields $E[f(\mathbf{X})] \leq E[f(\mathbf{Y})]$, as desired.

Concluding the main results in this section, we have Table 1.

\section{Applications and examples}

This section deals with applications of the previous results. One can obtain a series of probability and expectation inequalities for multivariate elliptical random variables. We will restrict ourselves to applications concerning the supermodular ordering.

\subsection{Slepian's theorem}

Slepian's theorem for multivariate normal distributions with non-singular covariance matrix can be found in Tong (1980). Das Gupta et al. (1972) generalized Slepian's theorem to the elliptical distributions with non-singular covariance matrix which was later proved in a different way by Joag-dev et al. (1983). Joe (1990) provided a shorter and elementary proof. For its extension to the case of singular covariance matrix the reader is referred to Fang and Liang (1989). Here we give a simple proof. Further results on the normal comparison inequalities of Slepian type can be found in Li and Shao (2002), Yan (2009) and Chernozhukov et al. (2015). 


\begin{tabular}{|} 
Table 1: Comparison criteria for $\mathbf{X} \sim E_{n}\left(\boldsymbol{\mu}^{x}, \boldsymbol{\Sigma}^{x}, \phi\right)$ and $\mathbf{Y} \sim E_{n}\left(\boldsymbol{\mu}^{y}, \boldsymbol{\Sigma}^{y}, \phi\right)$ \\
\begin{tabular}{|c||c|c|}
\hline Constraints on parameters & Relationship & Order \\
\hline $\boldsymbol{\mu}^{x} \leq \boldsymbol{\mu}^{y}, \boldsymbol{\Sigma}^{y}=\boldsymbol{\Sigma}^{x}$ & $\Leftrightarrow$ & $\mathbf{X} \leq_{s t} \mathbf{Y}$ \\
$\boldsymbol{\mu}^{x}=\boldsymbol{\mu}^{y}, \boldsymbol{\Sigma}^{y}-\boldsymbol{\Sigma}^{x} \succeq \mathbf{O}$ & $\Leftrightarrow$ & $\mathbf{X} \leq_{c x} \mathbf{Y}$ \\
$\boldsymbol{\mu}^{x}=\boldsymbol{\mu}^{y}, \boldsymbol{\Sigma}^{y}-\boldsymbol{\Sigma}^{x} \succeq \mathbf{O}$ & $\Leftrightarrow$ & $\mathbf{X} \leq_{l c x} \mathbf{Y}$ \\
$\boldsymbol{\mu}^{x} \leq \boldsymbol{\mu}^{y}, \boldsymbol{\Sigma}^{y}-\boldsymbol{\Sigma}^{x} \succeq \mathbf{O}$ & $\Rightarrow$ & $\mathbf{X} \leq_{i c x} \mathbf{Y}$ \\
$\boldsymbol{\mu}^{x} \leq \boldsymbol{\mu}^{y}, \boldsymbol{\Sigma}^{y}-\boldsymbol{\Sigma}^{x} \succeq \mathbf{O}, \operatorname{det}\left(\boldsymbol{\Sigma}^{y}-\boldsymbol{\Sigma}^{x}\right)=0$ & $\Leftrightarrow$ & $\mathbf{X} \leq_{i c x} \mathbf{Y}$ \\
$\boldsymbol{\mu}^{x}=\boldsymbol{\mu}^{y}, \sigma_{i i}^{x}=\sigma_{i i}^{y}, \sigma_{i j}^{x} \leq \sigma_{i j}^{y}$ & $\Leftrightarrow$ & $\mathbf{X} \leq_{s m} \mathbf{Y}$ \\
$\boldsymbol{\mu}^{x} \leq \boldsymbol{\mu}^{y}, \sigma_{i i}^{x}=\sigma_{i i}^{y}, \sigma_{i j}^{x} \leq \sigma_{i j}^{y}$ & $\Leftrightarrow$ & $\mathbf{X} \leq_{i s m} \mathbf{Y}$ \\
$\boldsymbol{\mu}^{x}=\boldsymbol{\mu}^{y}=\mathbf{0}, \sigma_{i i}^{x}=\sigma_{i i}^{y}, \sigma_{i j}^{x} \leq \sigma_{i j}^{y}$ & $\Leftrightarrow$ & $\mathbf{X} \leq_{i s m} \mathbf{Y}$ \\
$\boldsymbol{\mu}^{x}=\boldsymbol{\mu}^{y}, \sigma_{i j}^{x} \leq \sigma_{i j}^{y}, \forall i, j$ & $\Leftrightarrow$ & $\mathbf{X} \leq_{d c x} \mathbf{Y}$ \\
$\boldsymbol{\mu}^{x} \leq \boldsymbol{\mu}^{y}, \sigma_{i j}^{x} \leq \sigma_{i j}^{y}, \forall i, j$ & $\mathbf{X} \leq_{i d c x} \mathbf{Y}$ \\
$\boldsymbol{\mu}^{x} \leq \boldsymbol{\mu}^{y}, \sigma_{i i}^{x}=\sigma_{i i}^{y}, \sigma_{i j}^{x} \leq \sigma_{i j}^{y}$ & $\Leftrightarrow$ & $\mathbf{X} \leq_{u o} \mathbf{Y}$ \\
$\boldsymbol{\mu}^{x}=\boldsymbol{\mu}^{y}=\mathbf{0}, \sigma_{i i}^{x}=\sigma_{i i}^{y}, \sigma_{i j}^{x} \leq \sigma_{i j}^{y}$ & $\Leftrightarrow$ & $\mathbf{X} \leq_{u o} \mathbf{Y}$ \\
$\boldsymbol{\mu}^{x}=\boldsymbol{\mu}^{y}, \sigma_{i i}^{x} \leq \sigma_{i i}^{y}, \sigma_{i j}^{x}=\sigma_{i j}^{y}$ & $\Leftrightarrow$ & $\mathbf{X} \leq_{c c x} \mathbf{Y}$ \\
$\boldsymbol{\mu}^{x} \leq \boldsymbol{\mu}^{y}, \sigma_{i i}^{x} \leq \sigma_{i i}^{y}, \sigma_{i j}^{x}=\sigma_{i j}^{y}$ & $\Leftrightarrow$ & $\mathbf{X} \leq_{i c c x} \mathbf{Y}$ \\
$\boldsymbol{\mu}^{x}=\boldsymbol{\mu}^{y}, \boldsymbol{\Sigma}^{y}-\boldsymbol{\Sigma}^{x}$ is copositive & $\Leftrightarrow$ & $\mathbf{X} \leq_{c p} \mathbf{Y}$ \\
$\boldsymbol{\mu}^{x}=\boldsymbol{\mu}^{y}, \boldsymbol{\Sigma}^{y}-\boldsymbol{\Sigma}^{x}$ is completely copositive & $\Leftrightarrow$ & $\mathbf{X} \leq_{c o p} \mathbf{Y}$ \\
\hline
\end{tabular}
\end{tabular}

Theorem 4.1. Let $\mathbf{X} \sim E_{n}\left(\boldsymbol{\mu}^{x}, \boldsymbol{\Sigma}^{x}, \phi\right)$ and $\mathbf{Y} \sim E_{n}\left(\boldsymbol{\mu}^{y}, \boldsymbol{\Sigma}^{y}, \phi\right)$. If $\mathbf{X}$ and $\mathbf{Y}$ have the same marginals and $\sigma_{i j}^{x} \leq \sigma_{i j}^{y}$ for all $1 \leq i<j \leq n$, then

$$
P\left(X_{1} \leq a_{1}, \cdots, X_{n} \leq a_{n}\right) \leq P\left(Y_{1} \leq a_{1}, \cdots, Y_{n} \leq a_{n}\right)
$$

and

$$
P\left(X_{1}>a_{1}, \cdots, X_{n}>a_{n}\right) \leq P\left(Y_{1}>a_{1}, \cdots, Y_{n}>a_{n}\right)
$$

hold for every $\mathbf{a} \in \mathbb{R}^{n}$. Furthermore, the inequality is strict if $\sigma_{i j}^{x} \leq \sigma_{i j}^{y}$ for some $i, j$ and if the supports of $\mathbf{X}, \mathbf{Y}$ are unbounded.

Proof Using Theorem 3.4 and the implication $\mathbf{X} \leq_{s m} \mathbf{Y} \Rightarrow \mathbf{X} \leq_{u o} \mathbf{Y}$ and $\mathbf{X} \leq_{l o} \mathbf{Y}$ yield the desired result.

The following result is an immediate consequence of Theorem 4.1.

Corollary 4.1. Let $\mathbf{X} \sim E_{n}(\mathbf{0}, \boldsymbol{\Sigma}, \phi)$, then the probabilities $P\left(\min _{1 \leq i \leq n} g_{i}\left(X_{i}\right)>C\right)$ and $P\left(\max _{1 \leq i \leq n} g_{i}\left(X_{i}\right) \leq\right.$ $C)$ are increasing in each $\sigma_{i j}$, where $g_{i}: \mathbb{R} \rightarrow \mathbb{R}, i=1,2, \cdots, n$, are either all increasing or all decreasing.

\subsection{Moment Inequalities}

In this section we can easily derive various simple but useful inequalities for certain functions of multivariate elliptical random variables. The proofs are based on the results in Section 3, the most important result is the one on supermodular orders. We remark that supermodular functions play a 
significant role in applied fields, such as risk management, insurance, queueing, macroeconomic dynamics, optimization and game theory.

The following are some useful results and properties of supermodular functions. The proofs can be found in Bäuerle (1997), Topkis (1998), Christofides and Vaggelatou (2004) and Marshall and Olkin (2011, P. 219).

Lemma 4.1. (a) If $f$ is increasing and supermodular, then $\max \{f, c\}$ is supermodular for all $c \in \mathbb{R}$.

(b) If $f: \mathbb{R}^{n} \rightarrow \mathbb{R}$ is supermodular then the function $\psi$, defined by $\psi\left(x_{1}, x_{2}, \cdots, x_{n}\right)=f\left(g_{1}\left(x_{1}\right), \cdots, g_{n}\left(x_{n}\right)\right)$, is also supermodular, whenever $g_{i}: \mathbb{R} \rightarrow \mathbb{R}, i=1,2, \cdots, n$, are either all increasing or all decreasing.

(c) If $f_{i}$ is increasing (decreasing) on $\mathbb{R}^{1}$ for $i=1,2, \cdots, n$, then $f(x)=\min \left\{f_{1}\left(x_{1}\right), \cdots, f_{n}\left(x_{n}\right)\right\}=$ $-\max \left\{f_{1}\left(x_{1}\right), \cdots, f_{n}\left(x_{n}\right)\right\}$ is supermodular on $\mathbb{R}^{n}$.

(d) $H(\mathbf{x})=\left(\sum_{k=1}^{n} g_{i}\left(x_{i}\right)-t\right)^{+},\left(\prod_{k=1}^{n} g_{i}\left(x_{i}\right)-t\right)^{+}$are supermodular for any $t \geq 0$.

(e) If $f$ is monotonic supermodular and $g$ is increasing and convex, then $g \circ f$ is monotonic and supermodular.

(f) $H(\mathbf{x})=\prod_{k=1}^{n} \phi_{i}\left(x_{i}\right)$ is supermodular, where $\phi_{i}: \mathbb{R} \rightarrow \mathbb{R}, i=1,2, \cdots, n$, are either all increasing or all decreasing.

(g) The function $f(x)=\nu\left(x_{1}+\cdots+x_{n}\right)$ is supermodular, where $\nu$ is increasing convex.

(h) $H(\mathbf{x})=-\frac{1}{n-1} \sum_{i=1}^{n}\left(X_{i}-\bar{X}\right)^{2}$ is supermodular.

(i) The function $H(\mathbf{x})=\max _{1 \leq k \leq n} \sum_{i=1}^{k} X_{k}$ is supermodular and increasing.

The following three theorems are immediate consequence of Theorem 3.4 and Lemma 4.1.

Theorem 4.2. Let $f$ be a convex function on $(-\infty, \infty)$. Assume that $\mathbf{X} \sim E_{n}\left(\boldsymbol{\mu}^{x}, \boldsymbol{\Sigma}^{x}, \phi\right)$ and $\mathbf{Y} \sim$ $E_{n}\left(\boldsymbol{\mu}^{y}, \boldsymbol{\Sigma}^{y}, \phi\right)$. If $\mathbf{X}$ and $\mathbf{Y}$ have the same marginal and $\sigma_{i j}^{x} \leq \sigma_{i j}^{y}$ for all $1 \leq i<j \leq n$, then

$$
E f\left(g_{1}\left(X_{1}\right)+\cdots+g_{n}\left(X_{n}\right)\right) \leq E f\left(g_{1}\left(Y_{1}\right)+\cdots+g_{n}\left(Y_{n}\right)\right),
$$

where $g_{1}, \cdots, g_{n}$ are monotonic in the same direction. In particular,

$$
E\left(X_{1}^{3}+\cdots+X_{n}^{3}\right)^{2} \leq E\left(Y_{1}^{3}+\cdots+Y_{n}^{3}\right)^{2} .
$$

Theorem 4.3. Let $\mathbf{X} \sim E_{n}\left(\boldsymbol{\mu}^{x}, \boldsymbol{\Sigma}^{x}, \phi\right)$ and $\mathbf{Y} \sim E_{n}\left(\boldsymbol{\mu}^{y}, \boldsymbol{\Sigma}^{y}, \phi\right)$. If $\mathbf{X}$ and $\mathbf{Y}$ have the same marginals and $\sigma_{i j}^{x} \leq \sigma_{i j}^{y}$ for all $1 \leq i<j \leq n$.

(i) Assume that $f: \mathbb{R}^{n} \rightarrow \mathbb{R}$ is supermodular and $g_{i}: \mathbb{R} \rightarrow \mathbb{R}, i=1,2, \cdots, n$, are either all increasing or all decreasing. Then $\operatorname{Ef}\left(g_{1}\left(X_{1}\right), \cdots, g_{n}\left(X_{n}\right)\right) \leq E f\left(g_{1}\left(Y_{1}\right), \cdots, g_{n}\left(Y_{n}\right)\right)$.

(ii) Assume that $f$ is increasing and supermodular. Then $E \max \{f(\mathbf{X}), 0\} \leq E \max \{f(\mathbf{Y}), 0\}$.

Theorem 4.4. Assume that $\mathbf{X} \sim E_{n}\left(\boldsymbol{\mu}^{x}, \boldsymbol{\Sigma}^{x}, \phi\right)$ and $\mathbf{Y} \sim E_{n}\left(\boldsymbol{\mu}^{y}, \boldsymbol{\Sigma}^{y}, \phi\right)$. If $\mathbf{X}$ and $\mathbf{Y}$ have the same marginal and $\sigma_{i j}^{x} \leq \sigma_{i j}^{y}$ for all $1 \leq i<j \leq n$, then

(i) $E \prod_{k=1}^{n} \phi_{i}\left(X_{i}\right) \leq E \prod_{k=1}^{n} \phi_{i}\left(Y_{i}\right)$, where $\phi_{k}: \mathbb{R} \rightarrow \mathbb{R}$ are monotonic in the same direction.

(ii) $E \min \left\{f_{1}\left(X_{1}\right), \cdots, f_{n}\left(X_{n}\right)\right\} \leq E \min \left\{f_{1}\left(Y_{1}\right), \cdots, f_{n}\left(Y_{n}\right)\right\}$ and

$E \max \left\{f_{1}\left(X_{1}\right), \cdots, f_{n}\left(X_{n}\right)\right\} \geq E \max \left\{f_{1}\left(Y_{1}\right), \cdots, f_{n}\left(Y_{n}\right)\right\}$, where $f_{i}: \mathbb{R} \rightarrow \mathbb{R}, i=1,2, \cdots, n$, are either all increasing or all decreasing.

(iii) $E S_{x}^{2} \geq E S_{y}^{2}$, where

$$
S_{x}^{2}=\frac{1}{n-1} \sum_{i=1}^{n}\left(X_{i}-\bar{X}\right)^{2}
$$


(iv) If $f$ is a non-decreasing convex function, then

$$
E f\left(\max _{1 \leq k \leq n} \sum_{i=1}^{k} X_{k}\right) \leq E f\left(\max _{1 \leq k \leq n} \sum_{i=1}^{k} Y_{k}\right) .
$$

Example 4.1 (Equicorrelated elliptical variables) Let $\mathbf{X} \sim E_{n}\left(\boldsymbol{\mu}, \boldsymbol{\Sigma}^{x}, \phi\right)$ with $\boldsymbol{\Sigma}^{x}=\left(\sigma_{i j}^{x}\right)$ such that $\sigma_{i i}^{x}=\sigma^{2}, \sigma_{i j}^{x}=\rho_{x} \sigma^{2}$ for $1<i<j \leq n, \sigma^{2}>0, \rho_{x} \in[-1,1]$, and let $\mathbf{Y} \sim E_{n}\left(\boldsymbol{\mu}, \boldsymbol{\Sigma}^{y}, \phi\right)$ with $\boldsymbol{\Sigma}^{y}=\left(\sigma_{i j}^{y}\right)$ such that $\sigma_{i i}^{y}=\sigma^{2}, \sigma_{i j}^{y}=\rho_{y} \sigma^{2}$ for $1<i<j \leq n, \rho_{y} \in[-1,1]$. Then $\mathbf{X} \leq_{s m} \mathbf{Y}$ if and only if $\rho_{x} \leq \rho_{y}$. Bäuerle (1997) obtained the similar result for normal variables and $\rho_{x}, \rho_{y} \in[0,1]$. For any supermodular function $f: \mathbb{R}^{n} \rightarrow \mathbb{R}$, we deduce that the expectation $E f(\mathbf{X})$ is increasing in $\rho_{x}$. We remark that for this special correlated elliptical variable, the supermodularity of $f$ is not necessary. For example, if $f: \mathbb{R}^{n} \rightarrow \mathbb{R}$ is twice differentiable and satisfying $\frac{\partial^{2}}{\partial x_{i} \partial x_{j}} f(\mathbf{x}) \geq 0$ for $\mathbf{x} \in \mathbb{R}^{n}$ and for some $1 \leq i<j \leq n$, Proposition 1 in Joag-Dev, Perlman and Pitt (1983) and its Remarks on page 454 imply that $E f(\mathbf{X})$ is increasing in $\rho_{x}$. For example, if $\rho_{x} \leq \rho_{y}$, then $E\left(X_{1} X_{2} X_{3}^{2}\right) \leq E\left(Y_{1} Y_{2} Y_{3}^{2}\right)$ and $E\left(X_{1}^{3} X_{2}^{3} X_{3}^{4}\right) \leq E\left(Y_{1}^{3} Y_{2}^{3} Y_{3}^{4}\right)$.

Example 4.2 ( Serial correlated elliptical variables) Let $\mathbf{X} \sim E_{n}\left(\boldsymbol{\mu}, \boldsymbol{\Sigma}^{x}, \phi\right)$ and $\mathbf{Y} \sim E_{n}\left(\boldsymbol{\mu}, \boldsymbol{\Sigma}^{y}, \phi\right)$ with

$$
\boldsymbol{\Sigma}^{x}=\left(\begin{array}{cccc}
\sigma^{2} & \rho_{x} \sigma^{2} & \cdots & \rho_{x}^{n-1} \sigma^{2} \\
\rho_{x} \sigma^{2} & \sigma^{2} & \cdots & \rho_{x}^{n-2} \sigma^{2} \\
\vdots & \vdots & \ddots & \vdots \\
\rho_{x}^{n-1} \sigma^{2} & \rho_{x}^{n-2} \sigma^{2} & \cdots & \sigma^{2}
\end{array}\right), \boldsymbol{\Sigma}^{y}=\left(\begin{array}{cccc}
\sigma^{2} & \rho_{y} \sigma^{2} & \cdots & \rho_{y}^{n-1} \sigma^{2} \\
\rho_{y} \sigma^{2} & \sigma^{2} & \cdots & \rho_{y}^{n-2} \sigma^{2} \\
\vdots & \vdots & \ddots & \vdots \\
\rho_{y}^{n-1} \sigma^{2} & \rho_{y}^{n-2} \sigma^{2} & \cdots & \sigma^{2}
\end{array}\right)
$$

where $\sigma^{2}>0$ and $\rho_{x}, \rho_{y} \in[-1,1]$. Then $\mathbf{X} \leq_{s m} \mathbf{Y}$ if and only if $\rho_{x} \leq \rho_{y}$.

\section{Appendix 1.}

Theorem A1 Let $f(\mathbf{y})=\mathbf{y}^{\prime} \mathbf{A y}, \mathbf{y} \in \mathbb{R}^{n}$, where $\mathbf{A}$ is an $n \times n$ symmetric matrix. If $\mathbf{A z}=0$ has a positive solution, then $\mathbf{y}^{\prime} \mathbf{A y} \geq 0$ for all $\mathbf{y} \geq 0$ if and only if $\mathbf{y}^{\prime} \mathbf{A y} \geq 0$ for all $\mathbf{y} \in \mathbb{R}^{n}$.

Proof We prove the "only if" only. Since $f$ is quadratic, using Taylor's expansion we get

$$
f(\mathbf{x}+t \mathbf{y})=f(\mathbf{x})+t \mathbf{y}^{\prime} \nabla f(\mathbf{x})+t^{2} f(\mathbf{y}), \mathbf{x}, \mathbf{y} \in \mathbb{R}^{n}, t \in \mathbb{R},
$$

where $\nabla f(\mathbf{x})=2 \mathbf{A} \mathbf{x}$ is the gradient of $f$. We choose a $\mathbf{x}_{0}>0$ such that $\mathbf{A} \mathbf{x}_{0}=0$ and $f\left(\mathbf{x}_{0}\right)=0$. Then for any $\mathbf{y} \in \mathbb{R}^{n}$ and all sufficiently small positive $t$, one has $\mathbf{x}_{0}+t \mathbf{y} \geq 0$. Thus, $f\left(\mathbf{x}_{0}+t \mathbf{y}\right)=t^{2} f(\mathbf{y})$, and consequently $f(\mathbf{y}) \geq 0$.

\section{Appendix 2.}

Proof of Lemma 2.5. For $0 \leq \lambda \leq 1$, define

$$
\Psi_{\lambda}(\mathbf{t})=\exp \left(i \mathbf{t}^{\prime}\left(\lambda \boldsymbol{\mu}^{y}+(1-\lambda) \boldsymbol{\mu}^{x}\right) \psi\left(\mathbf{t}^{\prime}\left(\lambda \boldsymbol{\Sigma}^{y}+(1-\lambda) \boldsymbol{\Sigma}^{x}\right) \mathbf{t}\right), \mathbf{t} \in \mathbb{R}^{n} .\right.
$$

By using the Fourier inversion theorem

$$
\phi_{\lambda}(\mathbf{x})=\left(\frac{1}{2 \pi}\right)^{n} \int e^{-i \mathbf{t}^{\prime} \mathbf{x}} \psi_{\lambda}(\mathbf{t}) d \mathbf{t}
$$


The derivative of $\Psi_{\lambda}$ with respect to $\lambda$ is

$$
\begin{aligned}
\frac{\partial \Psi_{\lambda}(\mathbf{t})}{\partial \lambda}= & i \mathbf{t}^{\prime}\left(\boldsymbol{\mu}^{y}-\boldsymbol{\mu}^{x}\right) \exp \left(i \mathbf{t}^{\prime}\left(\lambda \boldsymbol{\mu}^{y}+(1-\lambda) \boldsymbol{\mu}^{x}\right) \psi\left(\mathbf{t}^{\prime}\left(\lambda \boldsymbol{\Sigma}^{y}+(1-\lambda) \boldsymbol{\Sigma}^{x}\right) \mathbf{t}\right)\right. \\
& +\mathbf{t}^{\prime}\left(\boldsymbol{\Sigma}^{y}-\boldsymbol{\Sigma}^{x}\right) \mathbf{t} \exp \left(i \mathbf{t}^{\prime}\left(\lambda \boldsymbol{\mu}^{y}+(1-\lambda) \boldsymbol{\mu}^{x}\right) \psi^{\prime}\left(\mathbf{t}^{\prime}\left(\lambda \boldsymbol{\Sigma}^{y}+(1-\lambda) \boldsymbol{\Sigma}^{x}\right) \mathbf{t}\right),\right.
\end{aligned}
$$

and hence,

$$
\begin{aligned}
\frac{\partial \phi_{\lambda}(\mathbf{t})}{\partial \lambda}= & \left(\frac{1}{2 \pi}\right)^{n} \int e^{-i \mathbf{t}^{\prime} \mathbf{x}} \frac{\partial \Psi_{\lambda}(\mathbf{t})}{\partial \lambda} d \mathbf{t} \\
= & \left(\frac{1}{2 \pi}\right)^{n} \int e^{-i \mathbf{t}^{\prime} \mathbf{x}} \Psi_{\lambda}(\mathbf{t}) i \mathbf{t}^{\prime}\left(\boldsymbol{\mu}^{y}-\boldsymbol{\mu}^{x}\right) d \mathbf{t} \\
& +\left(\frac{1}{2 \pi}\right)^{n} \int e^{-i \mathbf{t}^{\prime} \mathbf{x}} \mathbf{t}^{\prime}\left(\boldsymbol{\Sigma}^{y}-\boldsymbol{\Sigma}^{x}\right) \mathbf{t} \exp \left(i \mathbf{t}^{\prime}\left(\lambda \boldsymbol{\mu}^{y}+(1-\lambda) \boldsymbol{\mu}^{x}\right) \psi^{\prime}\left(\mathbf{t}^{\prime}\left(\lambda \boldsymbol{\Sigma}^{y}+(1-\lambda) \boldsymbol{\Sigma}^{x}\right) \mathbf{t}\right) d \mathbf{t}\right. \\
= & -\sum_{i=1}^{n}\left(\mu_{i}^{y}-\mu_{i}^{x}\right) \frac{\partial \phi_{\lambda}(\mathbf{t})}{\partial x_{i}}+\Delta,
\end{aligned}
$$

where

$$
\Delta=\left(\frac{1}{2 \pi}\right)^{n} \int e^{-i \mathbf{t}^{\prime} \mathbf{x}} \mathbf{t}^{\prime}\left(\boldsymbol{\Sigma}^{y}-\boldsymbol{\Sigma}^{x}\right) \mathbf{t} \exp \left(i \mathbf{t}^{\prime}\left(\lambda \boldsymbol{\mu}^{y}+(1-\lambda) \boldsymbol{\mu}^{x}\right) \psi^{\prime}\left(\mathbf{t}^{\prime}\left(\lambda \boldsymbol{\Sigma}^{y}+(1-\lambda) \boldsymbol{\Sigma}^{x}\right) \mathbf{t}\right) d \mathbf{t} .\right.
$$

Note that by (2.1), there exists a random variable $R \geq 0$ such that

$$
\psi\left(\mathbf{t}^{\prime} \mathbf{t}\right)=E\left(E\left(e^{i R \mathbf{t}^{\prime} \mathbf{U}^{(n)}} \mid R\right)\right)=\int_{0}^{\infty}{ }_{0} F_{1}\left(\frac{n}{2} ;-\frac{r^{2}\|\mathbf{t}\|^{2}}{4}\right) P(R \in d r),
$$

where

$$
{ }_{0} F_{1}(\gamma ; z)=\sum_{k=0}^{\infty} \frac{1}{(\gamma)_{k}} \frac{z^{k}}{k !} .
$$

Thus, for $u>0$,

$$
\begin{aligned}
\psi^{\prime}(u) & =\int_{0}^{\infty}{ }_{0} F_{1}\left(\frac{n}{2} ;-\frac{r^{2} u}{4}\right) P(R \in d r) \\
& =\int_{0}^{\infty} \frac{\partial}{\partial u}{ }_{0} F_{1}\left(\frac{n}{2} ;-\frac{r^{2} u}{4}\right) P(R \in d r) \\
& =-\frac{1}{2 n} \int_{0}^{\infty}{ }_{0} F_{1}\left(\frac{n}{2}+1 ;-\frac{r^{2} u}{4}\right) r^{2} P(R \in d r) \\
& \equiv-\frac{E\left(R^{2}\right)}{2 n} \psi_{1}(u),
\end{aligned}
$$

where

$$
\psi_{1}(u)=\frac{1}{E\left(R^{2}\right)} \int_{0}^{\infty}{ }_{0} F_{1}\left(\frac{n}{2}+1 ;-\frac{r^{2} u}{4}\right) r^{2} P(R \in d r)
$$

is a characteristic generator. Here

$$
c\left(\|\mathbf{t}\|^{2}\right):={ }_{0} F_{1}\left(\frac{n}{2}+1 ;-\frac{\|\mathbf{t}\|^{2}}{4}\right)
$$

is the characteristic function of uniform distribution in the unit sphere in $\mathbb{R}^{n}$. Thus, $\Delta$ can be rewritten as

$$
\Delta=\frac{E\left(R^{2}\right)}{2 n} \sum_{i=1}^{n} \sum_{j=1}^{n}\left(\sigma_{i j}^{y}-\sigma_{i j}^{x}\right) \frac{\partial^{2} \phi_{1 \lambda}(\mathbf{x})}{\partial x_{i} \partial x_{j}} .
$$


Define $g(\lambda)=\int_{\mathbb{R}^{n}} f(\mathbf{x}) \phi_{\lambda}(\mathbf{x}) d \mathbf{x}$, then $E[f(\mathbf{Y})]-E[f(\mathbf{X})]=g(1)-g(0)=\int_{0}^{1} g^{\prime}(\lambda) d \lambda$. The result follows since

$$
\begin{aligned}
g^{\prime}(\lambda)= & \int_{\mathbb{R}^{n}} f(\mathbf{x}) \frac{\partial \phi_{\lambda}(\mathbf{x})}{\partial \lambda} d \mathbf{x} \\
= & \int_{\mathbb{R}^{n}}\left(\boldsymbol{\mu}^{y}-\boldsymbol{\mu}^{x}\right)^{\prime} \nabla f(\mathbf{x}) \phi_{\lambda}(\mathbf{x}) d \mathbf{x} \\
& +\frac{E\left(R^{2}\right)}{2 n} \int_{\mathbb{R}^{n}} \operatorname{tr}\left\{\left(\boldsymbol{\Sigma}^{y}-\boldsymbol{\Sigma}^{x}\right) \mathbf{H}_{f}(\mathbf{x})\right\} \phi_{1 \lambda}(\mathbf{x}) d \mathbf{x} .
\end{aligned}
$$

Acknowledgements The author would like to thank Professor Xiaowen Zhou for helpful comments on an earlier draft of the paper. The research was supported by the National Natural Science Foundation of China (No. 11171179, 11571198, 11701319).

\section{References}

[1] Arlotto, A., Scarsini, M., 2009. Hessian orders and multinormal distributions. Journal of Multivariate Analysis 100, 2324-2330.

[2] Bäuerle, N., 1997. Inequalities for stochastic models via supermodular orderings. Communications in Statistics. Stochastic Models, 13(1), 181-201.

[3] Bäuerle, N., Bayraktar, E., 2014. A note on applications of stochastic ordering to control problems in insurance and finance. Stochastics: An International Journal of Probability and Stochastic Processes 86, 330-340.

[4] Bäuerle, N., Müller, A., 2006. Stochastic orders and risk measures: Consistency and bounds. Insurance: Mathematics and Economics 38, 132-148.

[5] Cambanis, S., Huang, S., Simons, G., 1981. On the theory of elliptically contoured distributions. Journal of Multivariate Analysis 11, 365-385.

[6] Cal, D., Carcamo, J., 2006. Stochastic orders and majorization of mean order statistics. Journal of Applied Probability 43, 704-712.

[7] Carter, M., 2001. Foundations of Mathematical Economics. Cambridge, Massachusetts 2001.

[8] Chernozhukov, V., Chetverikov, D., Kato, K., 2015. Comparison and anti-concentration bounds for maxima of Gaussian random vectors. Probab. Theory Relat. Fields 162, 47-70.

[9] Christofides, T.C., Vaggelatou, E., 2004. A connection between supermodular ordering and positive/negative association. Journal of Multivariate Analysis 88, 138-151.

[10] Cottle, R. W., Habetler, G. J., Lemke, C. E., 1970. Quadratic forms semi-definite over convex cones. In Proceed. of the Princeton Symposium on Mathematical Programming, pp. 551-565. Princeton Univ. Press, Princeton, 1970. 
[11] Das Gupta, S., Eaton, M.L., Olkin, I., Perlman, M.D., Savage, L.J., Sobel, M., 1972. Inequalities on the probability content of convex regions for elliptically contoured distributions. In: Sixth Berkeley Symposium on Probability and Statistics, II, pp. 241-265.

[12] Davidov, O., Peddada, S., 2013. The linear stochastic order and directed inference for multivariate ordered distributions. Ann. Statist. 41, 1-40.

[13] Denuit, M., Müller, A., 2002. Smooth generators of integral stochastic orders. Ann. Appl. Probab. $12,1174-1184$.

[14] Denuit, M., Dhaene, J., Goovaerts, M., Kaas, R., 2005. Actuarial Theory for Dependent Risks: Measures, Orders and Models. Wiley, New York.

[15] Ding, Y., Zhang, X., 2004. Some stochastic orders of Kotz-type distributions. Statist. Probab. Lett. 69, 389-396.

[16] El Karoui, N., 2009. Concentration of measure and spectra of random matrices: Applications to correlation matrices, elliptical distributions and beyond. The Annals of Applied Probability 19(6), $2362-2405$.

[17] Fábián, C. I., Mitra, G., Roman, D., 2011. Processing second-order stochastic dominance models using cutting-plane representations. Math. Program., Ser. A 130, 33-57.

[18] Fang, K. T., Liang, J. J., 1989. Inequalities for the partial sums of elliptical order statistics related to genetic selection. The Canadian Journal of Statistics 17(4), 439-446.

[19] Fang, K. W., Kotz, S., Ng, K. W., 1990. Symmetric Multivariate and Related Distributions. Chapman \& Hall.

[20] Fill, J. A., Kahn, J., 2013. Comparison inequalities and fastest-mixing markov chains. Annals of Applied Probability 23, 1778-1816.

[21] Gupta, A. K., Varga, T., Bodnar, T., 2013. Elliptically Contoured Models in Statistics and Portfolio Theory, 2nd ed. Springer, New York.

[22] Hazra, N. K., Kuiti, M. R., Finkelstein, M., Nanda, A. K., 2017. On stochastic comparisons of maximum order statistics from the location-scale family of distributions. Journal of Multivariate Analysis 160, 31-41.

[23] Houdré, C., Pérez-Abreu, V., Surgailis, D., 1998. Interpolation, correlation identities, and inequalities for infinitely divisible variables. J. Fourier Anal. Appl. 4, 651-668.

[24] Hu, T. Z., Zhuang, W. W., 2006. Stochastic orderings between p-spacings of generalized order statistics from two samples. Probability in the Engineering and Informational Sciences 20, 465-479.

[25] Joag-Dev, K., Perlman, M., Pitt, L., 1983. Association of normal random variables and Slepian's inequality. Ann. Probab. 11(2), 451-455.

[26] Joe, H., 1990. Multivariate concordance. J. Multivariate Anal. 35, 12-30. 
[27] Kelker, D., 1970. Distribution theory of spherical distributions and location-scale parameter generalization. Sankhy $\bar{a} 32,419-430$.

[28] Landsman, Z., Tsanakas, A., 2006. Stochastic ordering of bivariate elliptical distributions. Statistics \& Probability Letters 76, 488-494.

[29] Li, W, V., Shao, Q. M., 2002. A normal comparison inequality and its applications. Probab. Theory Relat. Fields 122, 494-508.

[30] López-Díaz, M. C., López-Díaz M., Martnez-Fernández, S., 2018. A stochastic order for the analysis of investments affected by the time value of money. Insurance: Mathematics and Economics 83, $75-82$.

[31] Marshall, A., Olkin, I., 1979. Inequalities: Theory of Majorization and its Applications. Academic Press, New York.

[32] Meester, L. E., Shanthikumar, J. G., 1993. Regularity of stochastic processes. Probability in the Engineering and Informational Sciences 7(3), 343-360.

[33] Müller, A., 1997. Stochastic orders generated by integrals: A unified study. Adv. Appl. Probab. 29 414-428.

[34] Müller, A., 2001. Stochastic ordering of multivariate normal distributions. Ann. Inst. Statist. Math. $53,567-575$

[35] Müller, A., Scarsini, M., 2000. Some remarks on the supermodular order. Journal of Multivariate Analysis 73, 107-119.

[36] Müller, A., Scarsini, M., 2006. Stochastic order relations and lattices of probability measures. SIAM Journal on Optimization 16, 1024-1043.

[37] Müller, A., Stoyan, D., 2002. Comparison Methods for Stochastic Models and Risks. John Wiley \& Sons Ltd., Chichester.

[38] Pan, X., Qiu, G., Hu, T., 2016. Stochastic orderings for elliptical random vectors. Journal of Multivariate Analysis 148, 83-88.

[39] Rüschendorf, L., 1980. Inequalities for the expectation of $\Delta$-monotone functions. Z. Wahrscheinlichkeitsth. verw. Gebiete 54, 341-349

[40] Scarsini, M., 1998. Multivariate convex orderings, dependence, and stochastic equality. J. Appl. Prob. 35, 93-103.

[41] Sha, X. Y., Xu, Z. S., Yin, C. C., 2019. Elliptical distribution-based weight-determining method for ordered weighted averaging operators. International Journal of Intelligent Systems 34, 858-877.

[42] Shaked, M., Shanthikumar, J.G., 1990. Parametric stochastic convexity and concavity of stochastic processes. Annals of the Institute of Statistical Mathematics 42: 509-531.

[43] Shaked, M., Shanthikumar, J. G., 1997. Supermodular stochastic orders and positive dependence of random vectors. J. Multivariate Anal. 61, 86-101. 
[44] Shaked, M., Shanthikumar, J.G., 2007. Stochastic Orders. Springer, New York.

[45] Tong, Y.L., 1980. Probability inequalities in Multivariate Distributions. Academic Press, New York.

[46] Topkis, D.M., 1998. Supermodularity and Complementarity. Princeton University Press, New Jersey.

[47] Whitt, W., 1986. Stochastic comparisons for non-Markov processes. Math. Operat. Res. 11, 608-618.

[48] Yan, L., 2009. Comparison inequalities for one sided normal probabilities. J. Theor. Probab. 22, $827-836$. 